\title{
Spin-Foams for All Loop Quantum Gravity
}

\author{
Wojciech Kamiński ${ }^{1}$, Marcin Kisielowski ${ }^{1}$, Jerzy Lewandowski ${ }^{1,2}$ \\ 1 Instytut Fizyki Teoretycznej, Uniwersytet Warszawski, \\ ul. Hoża 69, 00-681 Warszawa (Warsaw), Polska (Poland) \\ ${ }^{2}$ Institute for Gravitation and the Cosmos 85 Physics Department, \\ Penn State, University Park, PA 16802, U.S.A.
}

\begin{abstract}
The simplicial framework of Engle-Pereira-Rovelli-Livine spin-foam models is generalized to match the diffeomorphism invariant framework of loop quantum gravity. The simplicial spin-foams are generalized to arbitrary linear 2-cell spin-foams. The resulting framework admits all the spin-network states of loop quantum gravity, not only those defined by triangulations (or cubulations). In particular the notion of embedded spin-foam we use allows to consider knotting or linking spin-foam histories. Also the main tools as the vertex structure and the vertex amplitude are naturally generalized to arbitrary valency case. The correspondence between all the SU(2) intertwiners and the $\mathrm{SU}(2) \times \mathrm{SU}(2)$ EPRL intertwiners is proved to be 1-1 in the case of the Barbero-Immirzi parameter $|\gamma| \geq 1$, unless the co-domain of the EPRL map is trivial and the domain is non-trivial.
\end{abstract}

PACS numbers: 04.60.Pp 


\section{INTRODUCTION}

\section{A. Recent spin-foam models of gravitational field}

Spin-foams were introduced as histories of quantum spin-network states of loop quantum gravity [1] (LQG) by Reisenberger and Rovelli [2]. That idea gave rise to spin-foam models [4 6] (SFM) (see also Rovelli's book [1]). The spin-foam model of 3-dimensional gravity is derived from a discretization of the $\mathrm{BF}$ action (see [4] and the references therein). An alternative derivation of the spin-foam model from $2+1 \mathrm{LQG}$ was proposed by [5, 7]. For the 4-dimensional gravity there were several approaches [2, 13], however, the Barrett-Crane model [8] became the obligatory spinfoam model for almost 10 years. The actual relation between that model and LQG was derived in the seminal works of Reisenberger [11] and Baez [4]. The BC model is mathematically elegant, plays a role in mathematical physics and a priori there is no reason for that model to be wrong. Nonetheless, it was shown not to have sufficiently many degrees of freedom to ensure the correct classical limit [9]. That discovery produced an intensive research on suitable modification. Finally, two teams: ( $i$ ) Engle, Pereira, Rovelli and Livine and ( $i i$ ) Freidel and Krasnov found systematic derivations of a spin-foam model of gravitational field using as the starting point a discretization of the Holst action (the Palatini action plus an extra term which does not change the classical equations of motion is multiplied by an arbitrary real parameter whose inverse is named after Barbero and Immirzi [29]). The result of their effort is a new, consistent spin-foam model valid for the Barbero-Immirzi parameter belonging to the interval $-1 \leq \gamma \leq 1$, a promising candidate for a path integral formulation of LQG, and two different models for $|\gamma|>1$ [14 16] (see [16] for the comparison). The values of the Barbero-Immirzi parameter predicted by various black hole models belong to the former interval [17].

\section{B. The incompatibilities between LQG and some SFMs}

Although the correspondence between LQG and SFMs was settled down recently due to the EPRL model, there are still several incompatibilities between these frameworks.

The first difference can be found already at the conceptual level. Loop quantum gravity is a quantum theory of gravitational field with all its local degrees of freedom. This is not a discretized theory. The (kinematical) observables labelled by curves and 2-surfaces form a complete set for the continuum theory. The discreteness of LQG is a property of the quantum representation 18|. The SFMs on the other hand, are derived as quantizations of discretized classical theories [6, 13, 19, 20].

Secondly, LQG is a diffeomorphism invariant theory of fields on manifolds. The diffeomorphisms play a crucial role in LQG. They are responsible for the diversity of embedded graphs and spin-networks labelling the quantum states. Theories of discretized spacetime, on the other hand, including the EPRL model, seem to describe piecewise flat geometries defined on piecewise linear manifolds. In order to match them, either LQG should be restricted to the piecewise linear manifolds and piecewise linear spin-networks, or the SFMs should be suitably generalized.

The third difference would arise even if one restricted LQG to the piecewise linear spaces as suggested above. In the LQG canonical framework, whose quantum states are labelled by embedded spin-networks, there is no justified way to restrict the graphs to those dual to triangulations of the underlying 3-manifold. The EPRL model on the other hand, uses only simplicial complexes (or, recently cubulations [28]) and the spin-networks defined on their boundaries. Therefore it does not define a spin-foam history of a generic spin-network state of LQG. In particular, LQG admits knotted and linked graphs. The simplicial SFMs do not allow such states as well as they do not allow graphs with vertices more than 4 -valent.

In the literature there is a competition between efforts toward piecewise linearization of LQG 21, 22 on the one hand, or generalizations of the simplicial constructions to the diffeomorphism covariant diversity [2, 5, 13, 25]. We would like to join the latter direction.

\section{The goal of the paper}

Our aim is to redirect the development of the spin-foam models, and most importantly the EPRL model, to that extent, that they can be used to define spin-foam histories of an arbitrary 
spin network state of LQG. The notion of embedded spin-foam we use, allows to consider knotting or linking spin-foam histories. Since the knots and links may play a role in LQG, it is an advantage not to miss the chance of keeping those topological degrees of freedom in a spin-foam approach. Next, we characterize the structure of a general spin-foam vertex. We encode all the information about the vertex structure in the spin-network induced on the boundary of the neighborhood of a given vertex. The converse construction of a vertex from any given embedded spin-network was described by Reisenberger [13]. In the set of the spin-foams we define the generalized EPRL spin-foams. As an introduction to that important step, we formulate our definition of the $n$-valent Barrett-Crane intertwiner. That definition is merely equivalent to that of $[10-12]$, however we spell it out in our notation for the clarity and the sake of precision. Finally we generalize the Engle-Pereira-Rovelli-Livine intertwiner to the general spin-foams. A technical result concerns the correspondence between all the SU(2) intertwiners and the EPRL intertwiners. We show that the correspondence is 1-1 in the case of the Barbero-Immirzi parameter $\gamma \geq 1$, unless the co-domain of the EPRL map is trivial and the domain is non-trivial.

We were motivated to do this research by two works we want to acknowledge. The first one is Baez's introduction to SFM [4]. It is from that classic paper that we take the definition of linear 2-cell complex with boundaries and the definition of a spin-foam. The second paper we appreciate so much is the derivation of the new spin-foam model from the Holst action by Engle, Livine, Pereira and Rovelli [6]. Those two excellent works brought spin-foams closer to LQG. On the other hand, the works that should be and will be considered closer in the spirit of the current paper, are the Freidel-Krasnov model [15] (especially in the range of $\gamma$ in which that model does not overlap with EPRL). Also the pioneering works of Reisenberger [2, 11, 13] contain a lot of ideas that still have not been explored enough in the literature.

\section{THE HILBERT SPACE FOR THEORIES OF CONNECTIONS}

\section{A. The cylindrical functions and the measure}

Consider a manifold $\Sigma$, a compact Lie group $G$, its Lie algebra $\mathfrak{g}$, and the set $\mathcal{A}(\Sigma)$ of the Lie algebra $\mathfrak{g}$ valued differential one-forms (connections) on $\Sigma$.

A $G$ valued parallel transport function on $\mathcal{A}$ is defined by each finite curve $p$ in $\Sigma$, namely for every $A \in \mathcal{A}$,

$$
U_{p}(A):=\operatorname{Pexp} \int_{p}-A
$$

The parallel transport functions are used to define the cylindrical functions $\operatorname{Cyl}(\mathcal{A}(\Sigma))$. A cylindrical function $\Psi: \mathcal{A} \rightarrow \mathbb{C}$ is defined by a finite set $P$ of finite, oriented curves $p_{1}, \ldots, p_{n}$ in $\Sigma$ and by a continuous function $\psi: G^{n} \rightarrow \mathbb{C}$. The corresponding cylindrical function is

$$
\Psi(A):=\psi\left(U_{p_{1}}(A), \ldots, U_{p_{n}}(A)\right) .
$$

The space $\operatorname{Cyl}(\mathcal{A}(\Sigma))$ of cylindrical functions admits the sup-norm, and can be completed to a $\mathrm{C}^{*}$ algebra. Moreover, a natural diffeomorphism invariant integral is defined

$$
\operatorname{Cyl}(\mathcal{A}(\Sigma)) \ni \Psi \mapsto \int d \mu_{0}(A) \Psi(A)
$$

The idea that led to that integral was the following: if the differentiability class of the curves is suitably defined, then for every cylindrical function $\Psi \in \operatorname{Cyl}(\mathcal{A}(\Sigma))$, there is an embedded graph $\gamma^{(1)}=\left\{e_{1}, \ldots, e_{n}\right\}$ and a continuous function $\psi \in \mathrm{C}^{(0)}\left(G^{n}\right)$ such that

$$
\Psi(A)=\psi\left(U_{e_{1}}(A), \ldots, U_{e_{n}}(A)\right) .
$$

And the continuation of the idea was to define the integral as

$$
\int d \mu_{0}(A) \Psi(A):=\int d \mu_{\mathrm{H}}\left(g_{1}\right) \ldots d \mu_{\mathrm{H}}\left(g_{n}\right) \psi\left(g_{1}, \ldots, g_{n}\right) .
$$


Finally, one had to make sure, that the right-hand side was independent of the choice of the non-uniquely defined graph $\gamma^{(1)}$. Fortunately, it was. The resulting Hilbert space $\mathrm{L}^{2}\left(\mathcal{A}(\Sigma), \mu_{0}\right)$ serves as the Hilbert space for background independent quantum theories of (Poisson commuting) connections, in particular as the kinematical Hilbert space in which Quantum Geometry of loop quantum gravity is defined. This is how the graphs entered the old loop quantum gravity and gave it the current shape.

The gauge invariant cylindrical functions are easily identified as the functions given by closed loops. The subspace $\mathcal{H}_{\Sigma} \subset \mathrm{L}^{2}\left(\mathcal{A}(\Sigma), \mu_{0}\right)$ of the gauge invariant functions is defined by the functions of the form

$$
\Psi(A)=\psi\left(U_{\alpha_{1}(A)}, \ldots, U_{\alpha_{m}(A)}\right)
$$

where $\alpha_{1}, \ldots, \alpha_{m}$ are free generators of the first homotopy group of an embedded graph $\gamma^{(1)}$.

An orthonormal basis of $\mathcal{H}_{\Sigma}$ can be constructed by endowing the embedded graphs with the spin-network structures (see ЏB).

In the Hilbert space $\mathrm{L}^{2}\left(\mathcal{A}(\Sigma), \mu_{0}\right)$ we define a quantum representation for the classical variables $(A, E)$ where $A$ is the connection variable introduced above, and $E$ is a $\mathfrak{g}^{*}$ valued differential form and the Poisson bracket is

$$
\{f, h\}=\int_{\Sigma} \frac{\delta}{\delta A_{a}^{i}} f \frac{\delta}{\delta E_{i}^{a}} h-\int_{\Sigma} \frac{\delta}{\delta A_{a}^{i}} h \frac{\delta}{\delta E_{i}^{a}} f .
$$

In particular, we define the quantum flux operators

$$
\hat{E}(S)=\frac{1}{i} \int_{S} \frac{\delta}{\delta A_{a}^{i}}
$$

across a 2-surface $S \subset \Sigma$.

\section{B. Abstract spin-networks}

\section{Definition}

Given a compact group $G$, a $G$-spin-network is a triple $(\gamma, \rho, \iota)$, an oriented, piecewise linear 1-complex $\gamma$ (a graph) equipped with two colourings: $\rho$, and $\iota$ defined below (see also Fig 1).

- The colouring $\rho$, maps the set $\gamma^{(1)}$ of the 1-cells (edges) in $\gamma$ into the $\operatorname{set} \operatorname{Irr}(G)$ of the (equivalence classes of) irreducible representations of $G$. That is, to every edge $e$ we assign an irreducible representation $\rho_{e}$ defined in the Hilbert space $\mathcal{H}_{e}$,

$$
e \mapsto \rho_{e}
$$

- The colouring $\iota$ maps each vertex $v \in \gamma^{(0)}$ (where $\gamma^{(0)}$ denotes the set of 0 -cells of $\gamma$ ) into the subspace

$$
\operatorname{Inv}\left(\bigotimes_{e \text { incoming to } v} \rho_{e}^{*} \otimes \bigotimes_{e^{\prime} \text { outgoing from } v} \rho_{e^{\prime}}\right) \subset \bigotimes_{e^{\text {incoming to } v}} \mathcal{H}_{e}^{*} \otimes \bigotimes_{e^{\prime} \text { outgoing from } v} \mathcal{H}_{e^{\prime}}
$$

each $\mathcal{H}_{e}^{*}$ denotes the dual vector space to $\mathcal{H}_{e}$, and the subspace consists of all the invariant vectors. The map will be denoted

$$
v \mapsto \iota_{v}
$$




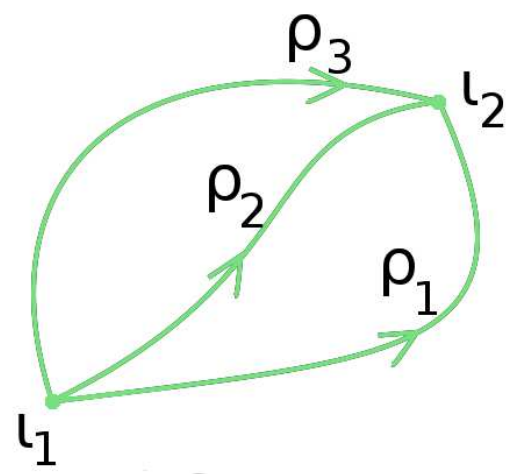

FIG. 1. A spin-network.

\section{Operations on spin-networks}

Flipping orientation is the first operation we consider. Given a spin-network $(\gamma, \rho, \iota)$ let a graph $\gamma^{\prime}$ be obtained by flipping the orientation of one of the edges, say $e_{1} \in \gamma^{(1)}$. The flipped orientation edge is denoted by $e_{1}^{-1}$. On $\gamma^{\prime}$ we can define a spin-network $\left(\gamma^{\prime}, \rho^{\prime}, \iota^{\prime}\right)$ using the data of $(\gamma, \rho, \iota)$ :

$$
\begin{aligned}
\iota^{\prime} & :=\iota \\
\rho_{e}^{\prime} & := \begin{cases}\left(\rho_{e_{1}}\right)^{*}, \quad e=e_{1}^{-1} \\
\rho_{e}, & \text { otherwise. }\end{cases}
\end{aligned}
$$

The complex conjugate spin-network $(\gamma, \bar{\rho}, \bar{\iota})$ to a given spin-network $(\gamma, \rho, \iota)$ is defined by using the conjugation map of any vector space

$$
V \ni v \mapsto \bar{v} \in \bar{V} .
$$

The conjugate vector space $\bar{V}$ is defined as the same set $V$ with a new multiplication ${ }^{-}$defined to be

$$
a^{-} v:=\bar{a} v,
$$

the same adding operation $\overline{+}=+$, and the conjugation map being the identity. Clearly,

$$
\bar{\iota}_{v}:=\overline{\iota_{v}}, \quad \bar{\rho}_{e}:=\overline{\rho_{e}} .
$$

The Hilbert conjugate spin-network $\left(\gamma^{\dagger}, \rho^{\dagger}, \iota^{\dagger}\right)$ to a spin-network $(\gamma, \rho, \iota)$ is a spin-network defined on the graph $\gamma^{\dagger}$ obtained by flipping the orientation of each of the edges of $\gamma$, and

$$
\begin{aligned}
\rho_{e^{-1}}^{\dagger} & :=\rho_{e}, \\
\iota_{v}^{\dagger} & :=\left(\iota_{v}\right)^{\dagger}
\end{aligned}
$$

where given the Hilbert space $\mathcal{H}$, we denote by

$$
\mathcal{H} \ni v \mapsto v^{\dagger} \in \mathcal{H}^{*}
$$

the antilinear map defined by the Hilbert product (that is " $|v\rangle^{\dagger}=\langle v|$ "). It is not hard to check, though, that each spin-network $\left(\gamma^{\dagger}, \rho^{\dagger}, \iota^{\dagger}\right)$ can be obtained from the complex conjugate spin-network $(\gamma, \bar{\rho}, \bar{\iota})$ by the operations of flipping orientation of each of the edges of $\gamma$.

Splitting an edge of a spin-network $(\gamma, \rho, \iota)$ consists of considering the graph $\gamma^{\prime}$ obtained from $\gamma$ by splitting one of its edges, say $e$ into

$$
e=e_{2}^{\prime} \circ e_{1}^{\prime},
$$


where we use a convention, that one first runs through $e_{1}^{\prime}$ and then through $e_{2}^{\prime}$. The edges $e_{1}^{\prime}, e_{2}^{\prime}$ are oriented in the agreement with $e$ and we insert the identity into a new vertex $v_{12}$ connecting the edges $e_{1}^{\prime}$ and $e_{2}^{\prime}$. In detail,

$$
\begin{aligned}
& \rho^{\prime}\left(e^{\prime}\right):=\left\{\begin{array}{l}
\rho(e), \text { if } e^{\prime}=e_{1}^{\prime}, e_{2}^{\prime} \\
\rho\left(e^{\prime}\right) \text { if } e^{\prime} \in \gamma^{(1)}
\end{array}\right. \\
& \iota^{\prime}\left(v^{\prime}\right):=\left\{\begin{array}{l}
\text { id, } \text { if } v^{\prime}=v_{12} \\
\iota\left(v^{\prime}\right) \text { if } v^{\prime} \in \gamma^{(0)}
\end{array}\right.
\end{aligned}
$$

\section{Spin-network functions}

With a spin-network $s=(\gamma, \rho, \iota)$ we associate a function 1

$$
\psi_{s}: G^{\gamma^{(1)}} \rightarrow \mathbb{C}
$$

Denote elements of $G^{\gamma^{(1)}}$ by

$$
g: \gamma^{(1)} \rightarrow G, \quad e \mapsto g_{e}
$$

For every $g \in G^{\gamma^{(1)}}$ there is a unique contraction

$$
\left.\psi_{s}(g):=\left(\bigotimes_{e \in \gamma^{(1)}} \rho_{e}\left(g_{e}\right)\right)\right\lrcorner\left(\bigotimes_{v \in \gamma^{(0)}} \iota_{v}\right) .
$$

In the abstract index notation, the contraction is defined as follows. For every edge $e \in \gamma^{(1)}$ we have $\rho_{B}^{A}\left(g_{e}\right)$ in (2.19). At the beginning point $v$ of $e$, there is an invariant $\iota_{v} \ldots A \ldots \ldots$ in (2.19) (the dots stand for the remaining indices) and at the end point $v^{\prime}$ of $e$, there is an invariant $\iota_{v^{\prime}} \ldots{ }^{\prime} \ldots$. The corresponding part of (2.19) reads

$$
\ldots \iota_{v} \ldots A \ldots \rho_{B}^{A}\left(g_{e}\right) \iota_{v^{\prime} \ldots} \ldots B \ldots \ldots
$$

Given a graph $\gamma$, the spin-network functions form naturally a basis of the Hilbert space $\mathcal{H}_{\gamma} \subset$ $L^{2}\left(G^{\gamma^{(1)}}, \mu_{\mathrm{H}}\right)$, where $\mu_{\mathrm{H}}$ is the Haar measure. The subspace $\mathcal{H}_{\gamma}$ coincides with the subspace of gauge invariant elements of $\mathrm{L}^{2}\left(G^{\gamma^{(1)}}, \mu_{\mathrm{H}}\right)$, where the gauge transformations are defined as follows: given a vertex $v \in \gamma^{(0)}$ the gauge transformation defined by $h \in G$ in $G^{\gamma^{(1)}}$ is

$$
(h, v) g_{e}=\left\{\begin{array}{l}
g_{e} h, \text { if } e \text { begins at } v \text { and ends elsewhere } \\
h^{-1} g_{e}, \text { if } e \text { ends at } v \text { and begins elsewhere } \\
h^{-1} g_{e} h, \text { if } e \text { begins and ends at } v \\
g_{e}, \text { otherwise. }
\end{array}\right.
$$

The general gauge transformation is defined by a sequence of elements of $G$ labelled by vertices.

Next, if a graph $\gamma^{\prime}$ is obtained by flipping the orientation in one of the edges, say $e_{0}$, of $\gamma$, then we define a map

$$
\begin{aligned}
G^{\gamma^{(1)}} & \rightarrow G^{\gamma^{\prime(1)}}, \\
g_{e} & \mapsto\left\{\begin{array}{l}
\left(g_{e_{0}^{-1}}\right)^{-1}, \text { if } e=e_{0} \\
g_{e}, \text { otherwise }
\end{array}\right.
\end{aligned}
$$

\footnotetext{
${ }^{1}$ Given two sets $X$ and $Y$, by $Y^{X}$ we denote the set of maps $X \rightarrow Y$. If $X$ has $n$ elements then $Y^{X} \sim Y^{n}$. This notation lets us avoid choosing an ordering in $X$.
} 
In the case when $\gamma^{\prime}$ is obtained by splitting one of the edges, say $e_{0}$, of $\gamma$ into $e=e_{2}^{\prime} \circ e_{1}^{\prime}$, the corresponding map is

$$
\begin{aligned}
f: G^{\gamma^{\prime(1)}} & \rightarrow G^{\gamma^{(1)}}, \\
f\left(g^{\prime}\right)_{e} & =\left\{\begin{array}{l}
g_{e_{2}^{\prime}} g_{e_{1}^{\prime}}, \text { if } e=e_{0} \\
g_{e}^{\prime}, \text { otherwise }
\end{array}\right.
\end{aligned}
$$

\section{Equivalence relation}

The spin-network functions define a natural equivalence relation in the space of spin-networks, namely two spin-networks are equivalent if and only if the corresponding spin-network functions are equal modulo the operations of splitting edges and/or reorienting edges accompanied by the maps (2.21) and, respectively, (2.23). Going back to the operations on the spin-networks of Sec. IB 2, we can see that splitting an edge of a spin-network and flipping the orientation map a spinnetwork into an equivalent one. Not surprisingly, the complex conjugation of a spin-network has the following interpretation in terms of the spin-network functions

$$
\psi_{\bar{s}}=\overline{\psi_{s}}
$$

The conclusion is, that modulo the equivalence relation we can change the orientation or split edges, not changing a given spin-network. This definition is also useful to define an equivalence of spin-foams.

\section{Evaluation of a spin-network}

Evaluation of a spin-network $s=(\gamma, \rho, \iota)$ is the number

$$
s \mapsto \psi_{s}(I)
$$

where $I \in G^{\gamma}$ is the identity element.

\section{The spin-network cylindrical functions of connections}

Finally, we are in a position to explain the application of the spin-networks in the Hilbert space of the cylindrical functions introduced in the previous section. Consider the 1-complexes (graphs) embedded in $\Sigma$, and embedded spin-networks defined on them. Given an embedded spin-network $s=(\gamma, \rho, \iota)$, we use the corresponding spin-network function $\psi_{s}$, to define a spin-network cylindrical function $\Psi_{s}: \mathcal{A} \rightarrow \mathbb{C}$. First, a connection $A \in \mathcal{A}$ defines an element $U_{\gamma}(A) \in G^{\gamma^{(1)}}$,

$$
U_{\gamma}(A): e \mapsto U_{e}(A) \in G
$$

Next, we use the spin-network function $\psi_{s}$,

$$
\Psi_{s}(A):=\psi_{s}\left(U_{\gamma}(A)\right)
$$

If we put a restriction on embeddings to be piecewise analytic then the spin-network cylindrical functions span a dense subset of the Hilbert space $\mathcal{H}_{\Sigma}$ and it is easy to construct from them an orthonormal basis. This assumption allow us to overcome some degenerated behavior of the smooth category.

The cylindrical functions lead to a natural equivalence relation between embedded spin-networks: two embedded spin-network states are equivalent if and only if the corresponding spin-network states are equal. 


\section{SPIN-FOAMS OF SPIN-NETWORKS AND OF SPIN-NETWORK FUNCTIONS}

\section{A. Classical motivation}

The motivation of the spin-foam approach to LQG is to develop an analog of the Feynman path integral. The idea of Rovelli and Reisenberger [2], is that the paths, should be suitably defined histories of the spin-network states. We address this issue in this section.

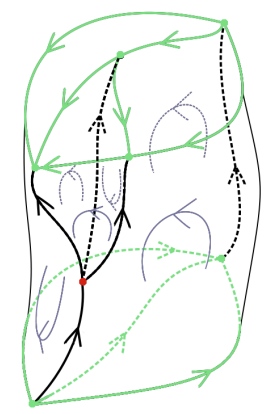

a)

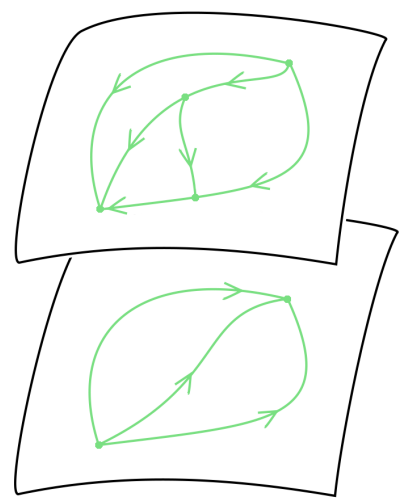

b)

FIG. 2. a) A history of a spin-network. b) The initial, and, respectively, final spin-network.

\section{B. Spin-foams}

\section{Foams}

By a foam we mean throughout this work an oriented linear 2-cell complex with (possibly empty) boundary. For the precise definition of the linear cell complexes we refer the reader to [4, 23]. Briefly, each foam $\kappa$ consists of 2-cells (faces), 1-cells (edges), and 0-cells (vertices).

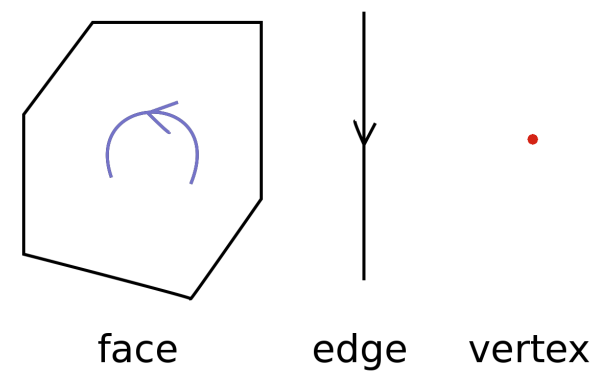

FIG. 3. Cells of the complex.

The faces are polygons, their sites are edges, the ends of the edges are vertices. Faces and edges are oriented, and the orientation of an edge is independent of the orientation of the face it is contained in. Each edge is contained in several (at least one) faces, each vertex is contained in several (at least one) edges.

The boundary $\partial \kappa$ is a 1-cell subcomplex (graph) of $\kappa$ such that there exists a one-to-one affine map $c: \partial \kappa \times[0,1] \rightarrow \kappa$ which maps each cell of $\partial \kappa \times[0,1]$ onto the unique cell of $\kappa$ and the set $\partial \kappa \times[0,1[$ onto an open subset of $\kappa$ (see appendix of [4] for details). An edge of $\kappa$ is an edge of the boundary if and only if it is contained in $\partial \kappa$. Otherwise, it is an internal edge. A vertex of $\kappa$ is a vertex of $\partial \kappa$ if and only if it is contained in exactly one internal edge of $\kappa$ (this is an important technical subtlety of the definition of a boundary). Otherwise, it is an internal vertex of $\kappa$. 


\section{Colouring}

Given a foam $\kappa$, a spin-foam is defined by introducing two colourings:

- $\rho$ colours the faces of $\kappa$ with irreducible representations of the group $G$ (the set of the faces of a foam $\kappa$ is denoted by $\left.\kappa^{(2)}\right)$,

$$
\begin{aligned}
\rho: \kappa^{(2)} & \rightarrow \operatorname{Irr}(G), \\
f & \mapsto \rho_{f}
\end{aligned}
$$

We consider representation $\rho_{f}$ as acting on a given Hilbert space $\mathcal{H}_{f}$.

- $\iota$ colours the internal edges of $\kappa$ (their set is denoted by $\kappa_{\text {int }}^{(1)}$ ) with invariants of suitable tensor product of the representations given by the colouring $\rho$. Let $e \in \kappa_{\text {int }}^{(1)}$. To define the space of invariants $\operatorname{Inv}_{e}$ we split the set of faces containing $e$, into faces whose orientation coincides with that of $e$, and, respectively, with the opposite orientation,

$$
\operatorname{Inv}_{e} \subset \bigotimes_{f \text { same orientation }} \mathcal{H}_{f} \otimes \bigotimes_{f^{\prime} \text { opposite orientation }} \mathcal{H}_{f^{\prime}}^{*}
$$

where the subset consist of the invariants of the representation

$$
\bigotimes_{f \text { same orientation }} \rho_{f} \otimes \bigotimes_{f^{\prime} \text { opposite orientation }} \rho_{f^{\prime}}^{*}
$$

The colouring $\iota$ is a map

$$
e \mapsto \iota_{e} \in \operatorname{Inv}_{e}
$$

a)

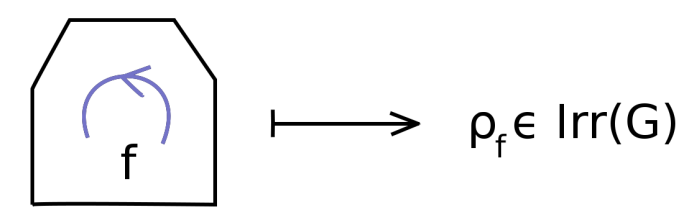

b)

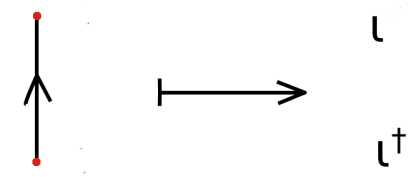

C)

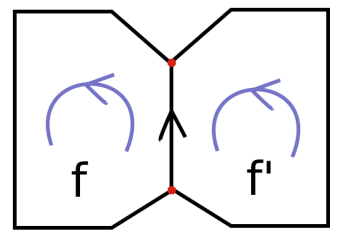

$\iota \in \operatorname{Inv}\left(\rho_{f}^{*} \otimes \ldots \otimes \rho_{f^{\prime}} \otimes \ldots\right)$

FIG. 4. a) Faces are coloured by irreducible representations of $G$. b), c) Edges are coloured with invariants. It is convenient to assign $\iota_{e}^{\dagger}$ to the beginning of $e$ and $\iota_{e}$ to the end of $e$.

Note that the spin-foam structure has been defined in the interior of a foam.

In fact, it is often convenient to think of $\iota_{e}^{\dagger}$ as assigned to the edge $e$ at the beginning point whereas at the end point we assign $\iota_{e}$.

\section{Induced boundary spin-network}

Given a spin-foam $(\kappa, \rho, \iota)$, the colourings $\rho$ and $\iota$ induce on the boundary $\partial \kappa$ a spin-network structure $(\partial \kappa, \partial \rho, \partial \iota)$. For every edge $e$ of $\partial \kappa$ let $f_{e}$ denote the unique face of $\kappa$ which contains $e$, 
and

$$
\partial \rho_{e}:= \begin{cases}\rho_{f_{e}}, & \text { if the orientations of } f_{e} \text { and } e \text { coincide } \\ \rho_{f_{e}}^{*}, & \text { if they are opposite. }\end{cases}
$$

For every vertex $v$ of $\partial \kappa$, let $e_{v}$ be the unique internal edge of $\kappa$ which contains $v$, and

$$
\partial \iota_{v}:=\left\{\begin{array}{l}
\iota_{e_{v}}^{\dagger}, \text { if } v \text { is the beginning of } e_{v} \\
\iota_{e_{v}} \text { if } v \text { is the end of } e_{v} .
\end{array}\right.
$$

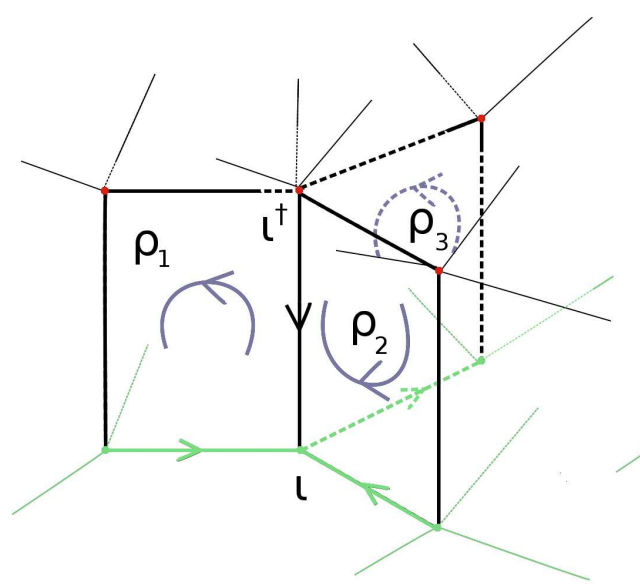

a)

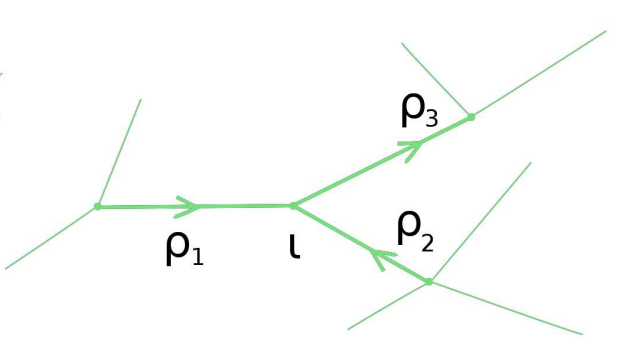

b)

FIG. 5. a) Spin-foam with boundary (in the bottom). b) Induced spin-network on the boundary.

\section{Operations on spin-foams}

Given a spin-foam $(\kappa, \rho, \iota)$ consider an oriented complex $\kappa^{\prime}$, obtained by flipping the orientation of one of the faces, say $f \in \kappa^{(2)}$. Denote the flipped orientation face by $f^{-1}$. On $\kappa^{\prime}$ we can define a spin-foam $\left(\kappa^{\prime}, \rho^{\prime}, \iota\right)$ modifying $\rho$,

$$
\rho_{f^{\prime}}^{\prime}:= \begin{cases}\left(\rho_{f}\right)^{*}, \quad f^{\prime}=f^{-1} \\ \rho_{f^{\prime}}, & \text { otherwise }\end{cases}
$$

Another operation involving a change of the orientation of a given spin-foam $(\kappa, \rho, \iota)$ is flipping the orientation of an edge $e$. Denote the resulting foam by $\kappa^{\prime}$. If $e$ is a boundary edge, we just leave the labellings $\rho$ and $\iota$ unchanged and consider the spin-foam $\left(\kappa^{\prime}, \rho, \iota\right)$. If $e$ is an internal edge, we modify $\iota$ as follows

$$
\iota_{e^{\prime}}^{\prime}:= \begin{cases}\left(\iota_{e}\right)^{\dagger}, & e^{\prime}=e^{-1} \\ \iota_{e^{\prime}}, & \text { otherwise. }\end{cases}
$$

In both cases presented above, (3.8) or (3.9), the resulting spin-foam will be considered equivalent to the starting $(\kappa, \rho, \iota)$. This equivalence allows to reorient spin-foams in the same class of equivalence.

Yet another operation is splitting a spin-foam $(\kappa, \rho, \iota)$, into two spin-foams $\left(\kappa_{1}, \rho_{1}, \iota_{1}\right)$ and $\left(\kappa_{2}, \rho_{2}, \iota_{2}\right)$ such that:

- $\kappa_{1}, \kappa_{2}$ are subcomplexes of $\kappa$ 
- the common part of $\kappa_{1}$ and $\kappa_{2}$ is a 1-subcomplex of the boundary of $\kappa_{1}$, as well as of the boundary of $\kappa_{2}$

The inverse operation operation to the splitting is called gluing of spin-foams.

\section{Spin-foam equivalence}

The equivalence relation introduced in the space of the spin-networks can be used to introduce equivalence relation between spin-foams. Spin-foams $(\kappa, \rho, \iota)$ and $\left(\kappa^{\prime}, \rho^{\prime}, \iota^{\prime}\right)$ are equivalent if the following two conditions are satisfied:

- there exists a bijective affine map (possibly not preserving orientation of the cells) from $\kappa$ onto $\kappa^{\prime}$

- for every splitting of $(\kappa, \rho, \iota)$ and the related splitting of $\left(\kappa^{\prime}, \rho^{\prime}, \iota^{\prime}\right)$, the corresponding spinnetworks induced on the boundaries are equivalent.

\section{Spin-foam trace}

\section{The vertex trace}

To every internal vertex of a spin-foam $(\kappa, \rho, \iota)$ we can naturally assign a number by contracting the invariants $\iota_{e} / \iota_{e^{\prime}}^{\dagger}$ which colour the incoming/outgoing edges. To begin with, given an internal vertex $v$ consider the tensor product

$$
\bigotimes_{\text {outgoing } e} \iota_{e}^{\dagger} \otimes \bigotimes_{\text {incoming } e^{\prime}} \iota_{e^{\prime}}
$$

For every face $f$ intersecting $v$, there are exactly two edges at $v$, say $e_{1}$ and $e_{2}$, contained in $f$. Take the corresponding invariants present in (3.10). One of them has exactly one index corresponding to the representation $\rho_{f}$. Then, the other one has exactly one index in the representation $\rho_{f}^{*}$. Contract those indices. And repeat the procedure for every face intersecting $v$. The result can be symbolically denoted by,

$$
\operatorname{Tr}\left(\bigotimes_{\text {outgoing } e} \iota_{e}^{\dagger} \otimes \bigotimes_{\text {incoming } e^{\prime}} \iota_{e^{\prime}}\right)=: \operatorname{Tr}_{v}(\kappa, \rho, \iota)
$$

and we call it the spin-foam vertex trace at a vertex $v$.

\section{The vertex spin-network}

The spin-foam vertex trace has a clear interpretation in terms of the spin-networks. Consider a spin-foam $(\kappa, \rho, \iota)$. Given an internal vertex $v$ and the intersecting faces, on each of the faces we consider a suitable neighbourhood of $v$. The neighbourhood is bounded by the segments of the sites of the face meeting at $v$, and a new extra edge connecting the segments (marked by a thinner curve on Fig. $7 \mathrm{a}$ )). The union of the resulting neighbourhoods of $v$ in each intersecting face is a foam neighbourhood of the vertex $v$. The foam neighbourhood of $v$ is a spin-foam with boundary itself. The boundary is formed by the thinner edges on Fig. 7]a). Denote the spin-network induced on the boundary (Fig. (7b)) by $s$, and the corresponding spin-network function by $\psi_{s}$. The vertex trace (3.11) of the spin-foam equals the evaluation of the conjugate spin-network

$$
\overline{\psi_{s}(I)}=\operatorname{Tr}_{v}(\kappa, \rho, \iota)
$$

The conjugate spin-network itself is the spin-network induced on the boundary of the spin-foam obtained from $(\kappa, \rho, \iota)$ by removing the neighbourhood of the vertex $v$. 


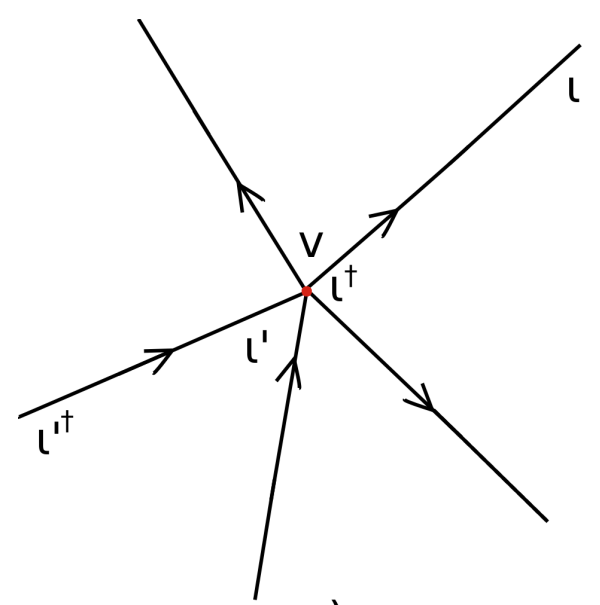

a)

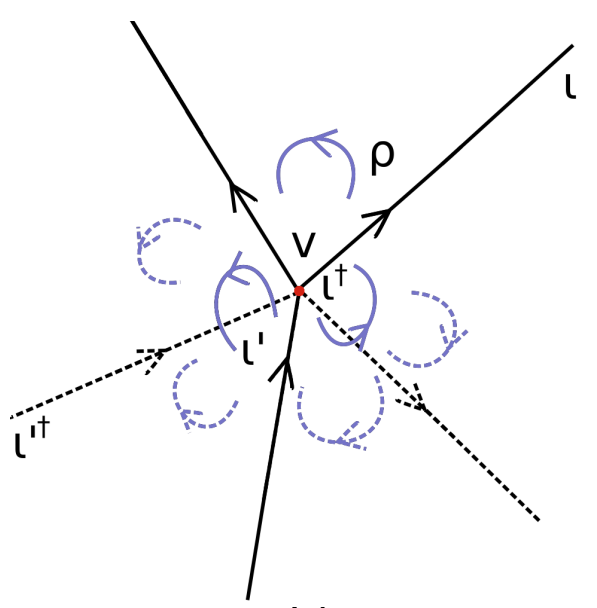

b)

FIG. 6. a) Edges meet at vertices. Every edge contributes an invariant or hermitian conjgate invariant. b) Every face meeting $v$ contains exactly two of the edges.

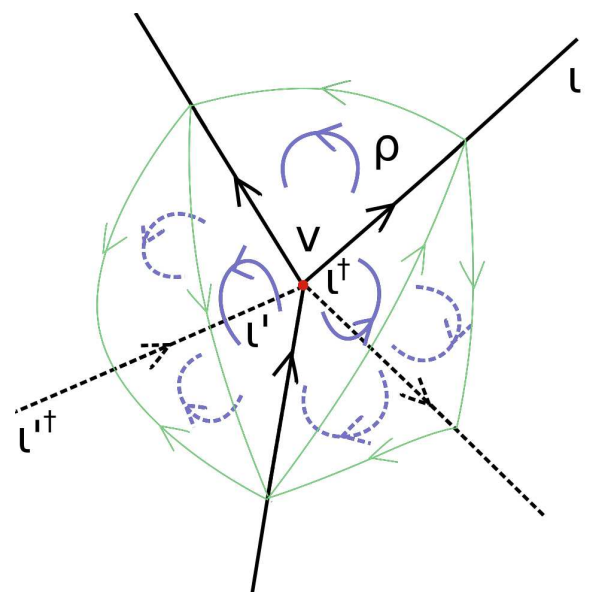

a)

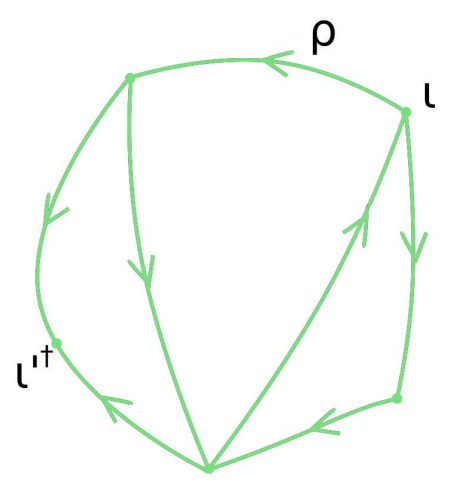

b)

FIG. 7. a) A foam neighbourhood of the vertex $v$ bounded by new, thin edges. b) The spin-network induced on the boundary of the neighbourhood. We call it: the vertex spin-network.

\section{The spin-foam trace}

The spin-foam trace of a spin-foam $(\kappa, \rho, \iota)$ will be defined by the product of the internal vertex traces. For the consistency with respect to the gluing, we introduce a factor for every vertex $v$ of the boundary of the spin-foam,

$$
\sqrt{\operatorname{Tr}\left(\partial \iota_{v}^{\dagger} \partial \iota_{v}\right)}
$$

where $(\partial \kappa, \partial \rho, \partial \iota)$ is the spin-network induced on the boundary and the Tr is the contraction of the interrelated indices. The spin-foam trace is defined to be

$$
\operatorname{Tr}(\kappa, \rho, \iota):=\prod_{\text {internal vertices } v} \operatorname{Tr}_{v}(\kappa, \rho, \iota) \prod_{\text {boundary vertices } v} \sqrt{\operatorname{Tr}\left(\partial \iota_{v}^{\dagger} \partial \iota_{v}\right)}
$$

If a spin-foam $(\kappa, \rho, \iota)$ is the result of the gluing of spin-foams $\left(\kappa_{1}, \rho_{1}, \iota_{1}\right)$, and $\left(\kappa_{2}, \rho_{2}, \iota_{2}\right)$ then

$$
\operatorname{Tr}(\kappa, \rho, \iota)=\operatorname{Tr}\left(\kappa_{1}, \rho_{1}, \iota_{2}\right) \operatorname{Tr}\left(\kappa_{2}, \rho_{2}, \iota_{2}\right) .
$$


Indeed, if a boundary vertex $v_{1}$ in $\left(\kappa_{1}, \rho_{1}, \iota_{1}\right)$ and a boundary vertex $v_{2}$ in $\left(\kappa_{2}, \rho_{2}, \iota_{2}\right)$ after the gluing become a single internal vertex $v$ of $(\kappa, \rho, \iota)$, then

$$
\operatorname{Tr}_{v}(\kappa, \rho, \iota)=\operatorname{Tr}\left(\partial \iota_{v_{1}}^{\dagger} \partial \iota_{v_{1}}\right)=\operatorname{Tr}\left(\partial \iota_{v_{2}}^{\dagger} \partial \iota_{v_{2}}\right)
$$

\section{Spin-foam of a spin-network function}

Thus far in this section we have been considering abstract spin-networks and abstract spinfoams. As we remember from the previous section, every spin-network state $\Psi_{s}$ is an element of the Hilbert space $\mathcal{H}_{\Sigma}$ of gauge invariant elements of the Hilbert space $\mathrm{L}^{2}\left(\mathcal{A}(\Sigma), d \mu_{0}\right)$ of the cylindrical functions of $G$-connections on $\Sigma$. The spin-network state is defined by a spin-network $s$ embedded in a manifold $\Sigma$.

There are two settings for considering spin-foams of spin-network states:

1. The causal approach [3 - 5] within which a spin-foam $(\kappa, \rho, \iota)$ is a history of an initial spinnetwork state $\Psi_{s_{\text {in }}} \in \mathcal{H}_{\Sigma_{\text {in }}}$ defined by a spin-network $s_{\text {in }}$ embedded in a manifold $\Sigma_{\text {in }}$. The history ends at a final spin-network state $\Psi_{s_{\text {out }}} \in \mathcal{H}_{\Sigma_{\text {out }}}$ defined by a spin-network $s_{\text {out }}$ embedded in $\Sigma_{\text {out }}$. The histories are defined by foams $\kappa$ embedded in a given manifold $M$ whose boundary is the disjoint sum

$$
\partial M=\Sigma_{\text {in }} \cup \Sigma_{\text {out }},
$$

and

$$
\partial \kappa \subset \Sigma_{\text {in }} \cup \Sigma_{\text {out }} .
$$

For each of the embedded spin-foams $(\kappa, \rho, \iota)$, the spin-network $s_{\text {in }}$ is the hermitian adjoint (= the complex conjugate) of the spin-network induced on $\partial \kappa \subset \Sigma_{\text {in }}$ whereas $s_{\text {out }}$ is the spin-network induced on $\partial \kappa \subset \Sigma_{\text {out }}$ (see explanation below).

2. The surface approach [1] (see Rovelli's book) and [24, 26], within which one considers a manifold $M$ and spin-network states $\Psi_{s} \in \mathcal{H}_{\Sigma}$ defined by the spin-networks embedded in

$$
\partial M=\Sigma .
$$

This approach is particularly natural in the Euclidean theory.

The emergence of the complex conjugation in the first item above, can be understood on the simplest example of the trivial spin-foam evolution (see Fig. [8]). Consider the product manifold

$$
M=\Sigma \times[0,1]
$$

and an embedded spin-network

$$
s_{\text {in }}=(\gamma, \rho, \iota)
$$

The spin-network $s$ induced on the boundary $\Sigma \times\{0\}$ is obtained from $s_{\text {in }}$ by flipping the orientations of the edges and taking the hermitian adjoint of its intertwiners, hence,

$$
s=\left(\gamma^{\dagger}, \rho^{\dagger}, \iota^{\dagger}\right)
$$

. Construct the "static" spin-foam history. The spin-foam faces are

$$
f=e \times[0,1]
$$

where $e$ runs through the set of the edges of $\gamma$, and the spin-foam edges are

$$
v \times[0,1],
$$

where $v$ runs through the set of the vertices of $\gamma$. Orient each face $e \times[0,1]$ consistently with the edge $e$ and each edge $v \times[0,1]$ according to the interval. Finally, the spin-network $s^{\prime}$ induced on 
the boundary $\Sigma \times\{1\}$ is obtained from $s$ by flipping the orientations of the edges and taking the hermitian adjoint of its intertwiners, hence,

$$
s^{\prime}=(\gamma, \rho, \iota)
$$

according to the definitions from Sec. ПB2, hence the corresponding state is

$$
\Psi_{s^{\prime}}=\overline{\Psi_{s_{\text {in }}}},
$$

provided we identify $\Sigma \times\{0\}$ with $\Sigma \times\{1\}$. According to the definition of the final spin-network state

$$
s_{\text {out }}=s^{\prime}=(\gamma, \rho, \iota)=s^{\dagger}=s_{\text {in }},
$$

in this static spin-foam case, as one would expect. There is a natural equivalence relation in the

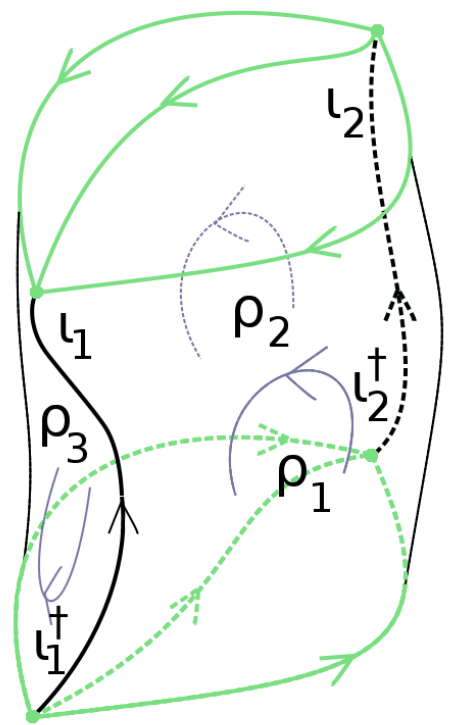

a)

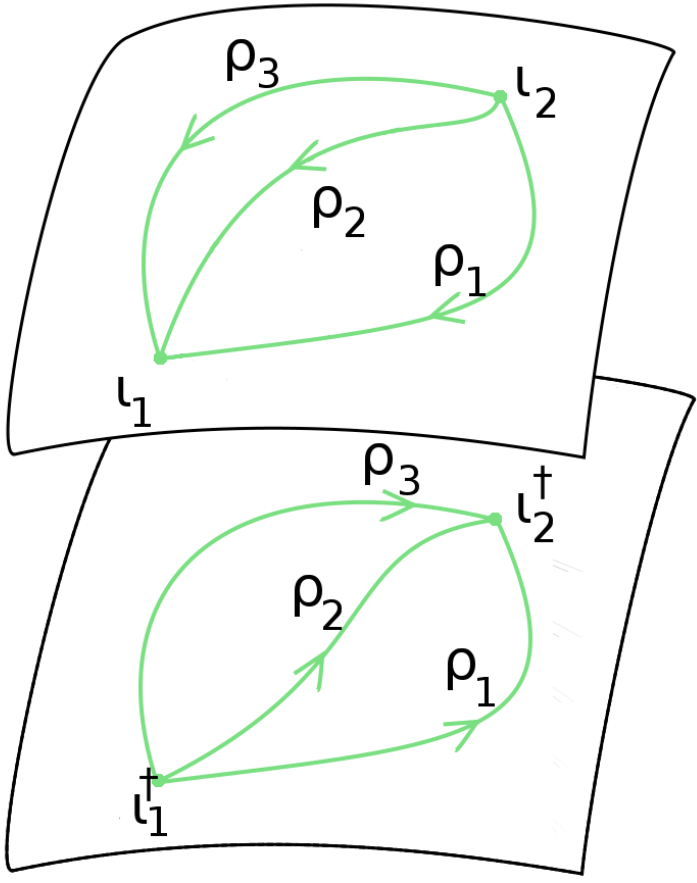

b)

FIG. 8. a) The static history of a spin-network state. b) The spin-network induced on the lower part of the boundary is the hermitian adjoint of the the initial (and at the same time final) spin-network.

space of embedded spin-foams in a given $\Sigma \times \mathbb{R}$. Given an embedded spin-foam $(\kappa, \rho, \iota)$, a generic cross section of $\Sigma \times \mathbb{R}$ cuts the spin-foam into two spin-foams with boundaries, and the spin-foam induces an embedded spin-network $s$ on the cross section (say, that we use the upper part of the spin-foam to induce $s$ ) and the corresponding spin-network state $\Psi_{s}$. Another embedded spin-foam is equivalent to $(\kappa, \rho, \iota)$ if it induces the same spin-network state on every generic cross section.

\section{THE SPIN-FOAM MODELS OF EUCLIDEAN 4-SPACETIME}

\section{A. Motivation}

The starting point is the gravity theory in terms of the Palatini action

$$
S_{\mathrm{P}}(e, A)=\frac{1}{2 \kappa} \int_{\Sigma \times \mathbb{R}} \frac{1}{2} \epsilon_{I J K L} e^{I} \wedge e^{J} \wedge F^{K L},
$$


where $\left(e^{1}, e^{2}, e^{3}, e^{4}\right)$ is an orthonormal tetrad of 1 -forms, $\left(A_{1}^{1}, A_{1}^{2}, \ldots, A_{4}^{4}\right)$ are connection 1 -forms which set an antisymmetric 1-form matrix

$$
A_{J}^{K} \eta_{K I}+A_{I}^{K} \eta_{K J}=0
$$

of the curvature

$$
F_{J}^{I}=d A_{J}^{I}+A_{K}^{I} \wedge A_{J}^{K}
$$

and the indices are raised and lowered with the matrix $\eta_{I J}$ which in the Euclidean case is $\delta_{I J}$. A classically irrelevant modification whose result is called the Holst action [27] and reads

$$
S_{\mathrm{H}}(e, A)=\frac{1}{2 \kappa} \int_{\Sigma \times \mathbb{R}} \frac{1}{2} \epsilon_{I J K L} e^{I} \wedge e^{J} \wedge F^{K L}+\frac{1}{\gamma} e^{I} \wedge e^{J} \wedge F_{I J},
$$

in fact leads to nonequivalent quantum theory depending on the value of the Barbero-Immirzi parameter $\gamma$. The theory can be interpreted as given by the action

$$
S(E, A)=\int_{\Sigma \times \mathbb{R}} E_{I J} \wedge F^{I J}
$$

with additional simplicity constraint

$$
E_{I J}=\frac{1}{2} \epsilon_{I J K L} e^{I} \wedge e^{J}
$$

in the Palatini case and

$$
E_{I J}=\frac{1}{2} \epsilon_{I J K L} e^{K} \wedge e^{L}+\frac{1}{2 \gamma} e_{I} \wedge e_{J}
$$

in the Holst action case.

\section{B. The scheme for embedded spin-foam models}

The recent spin-foam models of Barrett-Crane [8], Engle-Pereira-Rovelli-Livine [6] and FreidelKrasnov 15 fall into a common scheme. We combine it in below with the assumption that the spin-foams are histories of the embedded spin-network functions defined within the framework of the diffeomorphism invariant quantization of the theory of connections (see Section (II). The resulting scheme reads as follows:

- First, ignoring the simplicity constraints, one quantizes the theory 4.5) using the Hilbert space $\mathrm{L}^{2}\left(\mathcal{A}^{+}(\Sigma), \mathrm{d} \mu_{0}\right) \times \mathrm{L}^{2}\left(\mathcal{A}^{-}(\Sigma), \mathrm{d} \mu_{0}\right)$ of the cylindrical functions of the $\mathrm{SU}(2) \times \mathrm{SU}(2)$ connections on a 3 -surface $\Sigma$ and the subspace $\mathcal{H}_{\Sigma}^{+} \times \mathcal{H}_{\Sigma}^{-}$of the gauge invariant elements.

- Next, the simplicity constraints are suitably quantized to become linear quantum constraints imposed on the elements of $\mathcal{H}_{\Sigma}$. The constraint is locally defined at every vertex, hence it reduces to a constraint defined in each space $\operatorname{Inv}\left(\rho_{1} \otimes \ldots \rho_{k} \otimes \rho_{k+1}^{*} \otimes \ldots \otimes \rho_{N}^{*}\right)$ of the intertwiners. It defines a subspace $\operatorname{Inv}_{\text {Simp }}\left(\rho_{1} \otimes \ldots \rho_{k} \otimes \rho_{k+1}^{*} \otimes \ldots \otimes \rho_{N}^{*}\right)$ of simple intertwiners. That subspace may be nonempty only for some subset of representations. The spin-network functions defined by the simple intertwiners span the space of solutions to the simplicity constraint in $\mathcal{H}_{\Sigma}$.

- We consider the spin-foams of spin-network functions introduced in Section IIID with the intertwiners restricted to the spaces $\operatorname{Inv}_{\operatorname{Simp}}\left(\rho_{1} \otimes \ldots \rho_{k} \otimes \rho_{k+1}^{*} \otimes \ldots \otimes \rho_{N}^{*}\right)$ of simple intertwiners. That is, for every spin-foam $(\kappa, \rho, \iota)$ we consider, the map $\iota$ takes values in corresponding subspaces $\operatorname{Inv}_{\operatorname{Simp}}\left(\rho_{1} \otimes \ldots \rho_{k} \otimes \rho_{k+1}^{*} \otimes \ldots \otimes \rho_{N}^{*}\right)$.

- The amplitude of each spin-foam $(\kappa, \rho, \iota)$ is defined by using the spin-foam trace (3.14), namely

$$
\mathrm{A}(\kappa, \rho, \iota)=\left(\prod_{\text {intrinsic faces } f} \operatorname{dim}\left(\rho_{f}\right)\right) \operatorname{Tr}(\kappa, \rho, \iota)
$$


- To sum with respect to the spin-network histories with the amplitude as a weight, one fixes an orthonormal basis in each space $\operatorname{Inv}_{\operatorname{Simp}}\left(\rho_{1} \otimes \ldots \rho_{k} \otimes \rho_{k+1}^{*} \otimes \ldots \otimes \rho_{N}^{*}\right)$ of the simple intertwiners. In the (suitably defined) sums the intertwiners run through the fixed basis, for each choice of the representations at each vertex.

\section{Simple intertwiners}

There are three main proposals for the simple intertwiners:

1. that of Barrett-Crane (BC) corresponding to the Palatini action,

2. that of Engle-Pereira-Rovelli-Livine (EPRL) corresponding the Holst action with the value of the Barbero-Immirzi parameter $\gamma \neq \pm 1$,

3. that of Freidel-Krasnov (FK) also corresponding to the Holst action with the value of the Barbero-Immirzi parameter $\gamma \neq \pm 1$,

The BC intertwiners were derived by passing to the quantum level some symmetry between the self-dual and anti-self dual 2-forms. A few years ago it was noticed [9], that the BC states are insufficient to define physical semi-classical states of quantum gravity. That observation produced a new activity and led to the EPRL theory. The EPRL approach uses the Holst action. The simplicity constraint is systematically formulated and quantized. Its quantum solutions are derived. It is often stated that the failure of the $\mathrm{BC}$ approach consists in the too strong formulation of the quantum constraints. This is in fact not true. One can just rewrite the EPRL paper [6] replacing the Holst action by that of Palatini and repeating each argument and every calculation. The result will be the BC intertwiners. Hence, the real difference is in replacing the Palatini action by that of Holst. The FK model also uses the Holst action. The quantum simplicity constraint is encoded in a construction of suitable kinematical semiclassical states. Remarkably, the result coincides with the EPRL model as long as $0<\gamma<1$, whereas the result is different for $\gamma>1$. In particular $\gamma \rightarrow \infty$ is no longer the BC model [21].

Below we start with the definition of the $\mathrm{n}$-valent $\mathrm{BC}$ intertwiners which basically is equivalent to that of $10-12]$. Next we introduce the generalized EPRL intertwiners.

We do not study the FK intertwiners in the $\gamma>1$ in this paper.

\section{The $B C$ intertwiners}

The original BC formulation of the simplicity constraint relies on the fact that, given a 2-surface $S \subset \Sigma$, the metric area can be expressed in two equivalent ways by the self-dual / anti-self dual part

$$
E_{I J}^{ \pm}=\frac{1}{2}\left(E^{I J} \pm \epsilon_{I J K L} E^{K L}\right)
$$

of the frame field,

$$
\operatorname{Ar}_{S}\left(E^{+}\right)=\operatorname{Ar}_{S}\left(E^{-}\right)
$$

The corresponding quantum area operators are defined in the Hilbert space $\mathrm{L}^{2}\left(\mathcal{A}^{+}(\Sigma), \mathrm{d} \mu_{0}\right) \otimes$ $\mathrm{L}^{2}\left(\mathcal{A}^{-}(\Sigma), \mathrm{d} \mu_{0}\right)$, and take the form

$$
\operatorname{Ar}_{S}\left(\hat{E}^{+}\right)=\widehat{\operatorname{Ar}}_{S} \otimes 1, \quad \operatorname{Ar}_{S}\left(\hat{E}^{-}\right)=1 \otimes \widehat{\operatorname{Ar}}_{S},
$$

where $\widehat{\operatorname{Ar}}_{S}$ is the usual LQG area operator. The BC quantum simplicity constraint is

$$
\left(\widehat{\operatorname{Ar}}_{S} \otimes 1\right) \Psi=\left(1 \otimes \widehat{\operatorname{Ar}}_{S}\right) \Psi
$$

for every 2 -surface $S$. This set of equations labelled by various 2 -surfaces $S$, passes, via the spinnetwork cylindrical functions, to sets of equations defined in the spaces of the intertwiners between 
the tensor products of the representations of $\mathrm{SU}(2) \times \mathrm{SU}(2)$. It is sufficient to consider the invariants of the tensor products.

A representation $\rho_{j^{+} j^{-}}$of $\mathrm{SU}(2) \times \mathrm{SU}(2)$ is a pair of $\mathrm{SU}(2)$ representations $\left(\rho_{j^{+}}, \rho_{j^{-}}\right), j^{+}, j^{-}=$ $0, \frac{1}{2}, \ldots, \frac{n}{2}, \ldots$ acting in the tensor product $\mathcal{H}_{j^{+}} \otimes \mathcal{H}_{j^{-}}$

$$
\rho_{j^{+} j^{-}}\left(g^{+}, g^{-}\right)=\rho_{j^{+}}\left(g^{+}\right) \otimes \rho_{j^{-}}\left(g^{-}\right) .
$$

Given the tensor product $\rho_{j_{1}^{+} j_{1}^{-}} \otimes \ldots \otimes \rho_{j_{n}^{+} j_{n}^{-}}$the space of the invariants ( intertwiners of the tensor product of representations into the trivial representation) can be viewed as

$$
\begin{array}{r}
\operatorname{Inv}\left(\rho_{j_{1}^{+} j_{2}^{-}} \otimes \ldots \otimes \rho_{j_{n}^{+} j_{n}^{-}}\right)=\operatorname{Inv}\left(\rho_{j_{1}^{+}} \otimes \ldots \otimes \rho_{j_{n}^{+}}\right) \otimes \operatorname{Inv}\left(\rho_{j_{1}^{-}} \otimes \ldots \otimes \rho_{j_{n}^{-}}\right) \\
\subset \mathcal{H}_{j_{1}^{+}} \otimes \ldots \otimes \mathcal{H}_{j_{n}^{+}} \otimes \mathcal{H}_{j_{1}^{-}} \otimes \ldots \otimes \mathcal{H}_{j_{n}^{-}} .
\end{array}
$$

In each space $\operatorname{Inv}\left(\rho_{j_{1}} \otimes \ldots \otimes \rho_{j_{n}}\right)$ of the SU(2) intertwiners, the LQG area operators defined by all surfaces passing through the vertex induce a family of operators. There are only finitely many distinct among them. Slightly abusing the notation let us denote the induced area operators by $\widehat{\operatorname{Ar}}_{(1)}^{\rho}, \ldots, \widehat{\operatorname{Ar}}_{(m)}^{\rho}$, where $\rho=\left(\rho_{j_{1}}, \ldots, \rho_{j_{n}}\right)$. The BC condition passes now to

$$
\left.\widehat{\operatorname{Ar}}_{(I)}^{\left(\rho^{+}\right)} \otimes 1\right) \iota=\left(1 \otimes \widehat{\operatorname{Ar}}_{(I)}^{\left(\rho^{-}\right)}\right) \iota
$$

in the tensor product $\operatorname{Inv}\left(\rho_{j_{1}^{+}} \otimes \ldots \otimes \rho_{j_{n}^{+}}\right) \otimes \operatorname{Inv}\left(\rho_{j_{1}^{-}} \otimes \ldots \otimes \rho_{j_{n}^{-}}\right)$. Solutions to this equation can be called the $\mathrm{BC}$ intertwiners.

In the index notation the $\mathrm{BC}$ condition reads

$$
\widehat{\operatorname{Ar}}_{(I)}^{\left(\rho^{+}\right) A_{1}^{+} \ldots A_{n}^{+}} C_{1}^{+} \ldots C_{n}^{+} \iota^{C_{1}^{+} \ldots C_{n}^{+} A_{1}^{-} \ldots A_{n}^{-}}=\widehat{\operatorname{Ar}}_{(I)}^{\left(\rho^{-}\right) A_{1}^{-} \ldots A_{n}^{-}} C_{1}^{-} \ldots C_{n}^{-} \iota^{A_{1}^{+} \ldots A_{n}^{+} C_{1}^{-} \ldots C_{n}^{-}}
$$

Among the induced area operators there are $\widehat{\operatorname{Ar}}_{(I)}^{(\rho)}, I=1, \ldots, n$ (we have ordered them to be the first ones) such that

$$
\widehat{\operatorname{Ar}}_{(I)}^{\left(\rho^{+}\right)} \otimes 1=\sqrt{j_{I}^{+}\left(j_{I}^{+}+1\right)}(1 \otimes 1), \quad 1 \otimes \widehat{\operatorname{Ar}}_{(I)}^{\left(\rho^{-}\right)}=\sqrt{j_{I}^{-}\left(j_{I}^{-}+1\right)}(1 \otimes 1)
$$

It follows that a necessary condition for the existence of a $\mathrm{BC}$ intertwiner in a given intertwiner space is

$$
j_{1}^{+}=j_{1}^{-}, \quad j_{n}^{+}=j_{n}^{-} .
$$

Hence, each $\mathrm{SU}(2) \times \mathrm{SU}(2)$ intertwiner space which contains a $\mathrm{BC}$ intertwiner has the form

$$
\operatorname{Inv}\left(\rho_{j_{1}} \otimes \ldots \otimes \rho_{j_{n}}\right) \otimes \operatorname{Inv}\left(\rho_{j_{1}} \otimes \ldots \otimes \rho_{j_{n}}\right) .
$$

Now, the BC condition is (we drop the decoration $\rho$ of the operators, because now it is the same on the left and on the right-hand side)

$$
\left(\widehat{\operatorname{Ar}}_{(I)} \otimes 1\right) \iota=\left(1 \otimes \widehat{\operatorname{Ar}}_{(I)}\right) \iota
$$

or in the index notation

$$
\widehat{\operatorname{Ar}}_{(I)}^{A_{1}^{+} \ldots A_{n}^{+}}{ }_{C}^{+} \ldots C_{n}^{+} \iota^{C_{1}^{+} \ldots C_{n}^{+} A_{1}^{-} \ldots A_{n}^{-}}=\widehat{\operatorname{Ar}}_{(I)}^{A_{1}^{-} \ldots A_{n}^{-}} C_{1}^{-} \ldots C_{n}^{-} \iota^{A_{1}^{+} \ldots A_{n}^{+} C_{1}^{-} \ldots C_{n}^{-}}
$$

This induced BC condition means that $\iota$ is a bilinear 2-form, the operators $\widehat{\operatorname{Ar}}_{(I)}$ are symmetric with respect to.

On the other hand, there is a distinguished bilinear form, which is an element of the intertwiner space at the

$$
\operatorname{Inv}\left(\rho_{j_{1}} \otimes \ldots \otimes \rho_{j_{n}}\right) \otimes \operatorname{Inv}\left(\rho_{j_{1}} \otimes \ldots \otimes \rho_{j_{n}}\right)
$$


defined by the unique invariants

$$
\epsilon_{j} \in \operatorname{Inv}\left(\rho_{j} \otimes \rho_{j}\right) .
$$

The normalization and reality condition reduce the rescaling ambiguity to \pm 1 . The product gives a distinguished non-invariant element

$$
\epsilon:=\epsilon_{j_{1}} \otimes \ldots \otimes \epsilon_{j_{n}} \in \mathcal{H}_{j_{1}} \otimes \ldots \otimes \mathcal{H}_{j_{n}} \otimes \mathcal{H}_{j_{1}} \otimes \ldots \otimes \mathcal{H}_{j_{n}},
$$

and the projection onto the intertwiner space,

$$
P: \mathcal{H}_{j_{1}} \otimes \ldots \otimes \mathcal{H}_{j_{n}} \rightarrow \operatorname{Inv}\left(\rho_{j_{1}} \otimes \ldots \otimes \rho_{j_{n}}\right)
$$

gives the distinguished intertwiner,

$$
\iota_{\mathrm{BC}}:=(P \otimes P) \epsilon .
$$

Now, let us see that $\iota_{\mathrm{BC}}$ satisfies the induced $\mathrm{BC}$ constraint (4.18). Every operator $L$ in $\operatorname{Inv}\left(\rho_{j_{1}} \otimes \ldots \otimes \rho_{j_{n}}\right)$ that commutes with the projection $P$, and is symmetric with respect to the bilinear form $\epsilon$ satisfies

$$
(L \otimes 1)(P \otimes P) \epsilon=(P \otimes P)(L \otimes 1) \epsilon=(P \otimes P)(1 \otimes L) \epsilon=(1 \otimes L)(P \otimes P) \epsilon .
$$

In particular the induced area operators have those properties, hence indeed $\iota_{\mathrm{BC}}$ does satisfy the $\mathrm{BC}$ condition (4.18).

The only thing one should check is that the intertwiner $\iota_{\mathrm{BC}}$ is not zero. The non-vanishing of $\iota_{\mathrm{BC}}$ whenever the $\mathrm{SU}(2)$ intertwiner space $\operatorname{Inv}\left(\rho_{j_{1}} \otimes \ldots \otimes \rho_{j_{n}}\right)$ is not empty itself, follows from the nondegeneracy of the invariant antisymmetric bilinear form $\epsilon_{\frac{1}{2}}$ which implies the nondegeneracy of each $\epsilon_{j}$, as well as the nondegeneracy of the product $\epsilon$. The non-vanishing of the projection onto the intertwiner subspace is the result of the identity

$$
(1 \otimes P) \epsilon=(P \otimes P) \epsilon=(P \otimes 1) \epsilon .
$$

In words, the identity means that if we apply the projection once, that is consider say $(1 \otimes P) \epsilon$, then the result is already an invariant with respect to the action of $\rho_{j_{1}} \otimes \ldots \otimes \rho_{j_{n}}$ in the first factor of the tensor product. Perhaps it is easier to see the non-vanishing in the index notation. According to the identity,

$$
\iota_{\mathrm{BC}}{ }^{A_{1} \ldots A_{n} B_{1} \ldots B_{n}}=P^{A_{1} \ldots A_{n}} C_{1} \ldots C_{n} \epsilon_{j_{1}}^{C_{1} B_{1}} \ldots \epsilon_{j_{n}}^{C_{n} B_{n}} .
$$

But the raising of each index $B_{i}$ with $\epsilon_{j_{i}}$ can not kill any nonzero tensor, in particular the projection $P$. (One may briefly say that $\iota_{\mathrm{BC}}$ is the identity operator $\operatorname{Inv}\left(\rho_{j_{1}} \otimes \ldots \otimes \rho_{j_{n}}\right) \rightarrow$ Inv $\left(\rho_{j_{1}} \otimes \ldots \otimes \rho_{j_{n}}\right)$ with the lower indices raised by the invariant bilinear form $\epsilon$.) Remarkably, if we rewrite the EPRL paper [6] for the Palatini action (rather the that of Holst considered by EPRL), we will derive for every intertwiner space $\operatorname{Inv}\left(\rho_{j_{1}} \otimes \ldots \otimes \rho_{j_{n}}\right) \otimes \operatorname{Inv}\left(\rho_{j_{1}} \otimes \ldots \otimes \rho_{j_{n}}\right)$ a unique solution. This solution will be exactly our very $\iota_{\mathrm{BC}}$ (see also below).

\section{The EPRL intertwiners}

The simplicity constraint (4.7) defined by the Holst action depends on a real parameter $\gamma \neq 0$, known as the Barbero-Immirzi parameter [29]. As in the BC case, a quantization of the Holst simplicity constraint passes to the intertwiner spaces

$$
\operatorname{Inv}\left(\rho_{j_{1}^{+} j_{2}^{-}} \otimes \ldots \otimes \rho_{j_{n}^{+} j_{n}^{-}}\right)=\operatorname{Inv}\left(\rho_{j_{1}^{+}} \otimes \ldots \otimes \rho_{j_{n}^{+}}\right) \otimes \operatorname{Inv}\left(\rho_{j_{1}^{-}} \otimes \ldots \otimes \rho_{j_{n}^{-}}\right) .
$$

According to the EPRL quantum simplicity constraint a necessary condition for the existence of a solution in a given intertwiner space is

$$
j_{I}^{+}=\frac{|\gamma+1|}{|\gamma-1|} j_{I}^{-}, \quad I=1, \ldots, n .
$$


An ingredient of the EPRL intertwiner is an SU(2) invariant

$$
\mathcal{I} \in \operatorname{Inv}\left(\rho_{k_{1}} \otimes \ldots \otimes \rho_{k_{n}}\right),
$$

where, to every pair $j_{I}^{+}, j_{I}^{-}$such that (4.28) the EPRL quantum constraint condition boils down to selecting a representation $\rho_{k_{I}}$ of $\mathrm{SU}(2)$, of the spin $k_{I}$ adjusted as follows

$$
k_{I}:=\left\{\begin{array}{l}
j^{+}+j^{-}, \quad \text { if }-1<\gamma<1 \\
\left|j^{+}-j^{-}\right|, \quad \text { if } \gamma<-1 \text { or } 1<\gamma .
\end{array}\right.
$$

Given $\mathcal{I}$, an EPRL intertwiner $\iota_{\operatorname{EPRL}}(\mathcal{I})$ is defined using the invariants

$$
c_{I} \in \operatorname{Inv}\left(\rho_{j_{I}^{+}} \otimes \rho_{j_{I}^{-}} \otimes \rho_{k_{I}}^{*}\right), \quad I=1, \ldots, n
$$

unique for every $I=1, \ldots, n$, modulo a factor (the normalization and reality can restrict the ambiguity to \pm 1$)$. First, construct a non-invariant element of $\mathcal{H}_{j_{1}^{+}} \otimes \ldots \otimes \mathcal{H}_{j_{n}^{+}} \otimes \mathcal{H}_{j_{1}^{-}} \otimes \ldots \otimes \mathcal{H}_{j_{n}^{-}}$, namely

$$
\left.c_{1} \otimes \ldots \otimes c_{n}\right\lrcorner \mathcal{I}
$$

that is, in the index notation

$$
c_{1}{ }^{A_{1}^{+} A_{1}^{-}}{ }_{B_{1}} \ldots c_{n}^{A_{n}^{+} A_{n}^{-}}{ }_{B_{n}} \mathcal{I}^{B_{1} \ldots B_{n}} .
$$

Next, use the projections

$$
P^{ \pm}: \mathcal{H}_{j_{1}^{ \pm}} \otimes \ldots \otimes \mathcal{H}_{j_{n}^{ \pm}} \rightarrow \operatorname{Inv}\left(\rho_{j_{1}^{ \pm}} \otimes \ldots \otimes \rho_{j_{n}^{ \pm}}\right)
$$

that is define

$$
\left.\iota_{\mathrm{EPRL}}(\mathcal{I}):=\left(P^{+} \otimes P^{-}\right) c_{1} \otimes \ldots \otimes c_{n}\right\lrcorner \mathcal{I} .
$$

This formula can be written in a simpler way by skipping one of the projections, namely

$$
\left.\left.\iota_{\operatorname{EPRL}}(\mathcal{I})=\left(P^{+} \otimes 1\right) c_{1} \otimes \ldots \otimes c_{n}\right\lrcorner \mathcal{I}=\left(1 \otimes P^{-}\right) c_{1} \otimes \ldots \otimes c_{n}\right\lrcorner \mathcal{I},
$$

and in the index notation

$$
\iota_{\operatorname{EPRL}}(\mathcal{I})^{A_{1}^{+} \ldots A_{n}^{+} A_{1}^{-} \ldots A_{n}^{-}}=P^{+{ }_{1}^{+} \ldots A_{n}^{+}} c_{D_{1}^{+} \ldots D_{n}^{+}}^{D_{1}^{D_{1}^{+}} A_{1}^{-}} B_{1} \ldots c_{n}{ }^{D_{n}^{+} A_{n}^{-}} B_{n} \mathcal{I}^{B_{1} \ldots B_{n}}
$$

As in the $\mathrm{BC}$ case, one can briefly describe that definition by saying that each lower index of the projection $P^{ \pm}$is raised by the bilinear form $c_{1} D_{1}^{+} A_{1}^{-} B_{1} \ldots c_{n} D_{n}^{+} A_{n}^{-} B_{n} \mathcal{I}^{B_{1} \ldots B_{n}}$.

Notice, that in the limit $\gamma \rightarrow \pm \infty$ the conditions (4.2814.2914.304.314.36) go to (see Section IV CD

$$
\begin{gathered}
j_{I}^{+}=j_{I}^{-}, \quad k_{I}=0, c_{I}=\epsilon_{j_{I}}, \mathcal{I}=1 \\
\iota_{\mathrm{EPRL}}(1)^{A_{1}^{+} \ldots A_{n}^{+} A_{1}^{-} \ldots A_{n}^{-}}:=P^{+A_{1}^{+} \ldots A_{n}^{+}} \epsilon_{j_{1}} D_{1}^{+} \ldots A_{n}^{-} \ldots \epsilon_{j_{n}} D_{n}^{+} A_{n}^{-}=\iota_{\mathrm{BC}} A_{1}^{+} \ldots A_{n}^{+} A_{1}^{-} \ldots A_{n}^{-}
\end{gathered}
$$

That is, the EPRL intertwiner becomes the BC intertwiner $\iota_{\mathrm{BC}}$. This is consistent with the fact that the classical Holst action becomes the Palatini action in the limit $\gamma \rightarrow \pm \infty$.

In practical calculations, the projector (4.22) can be written in terms of any basis

$$
\iota_{1}, \ldots, \iota_{N} \in \operatorname{Inv}\left(\rho_{j_{1}} \otimes \ldots \otimes \rho_{j_{n}}\right),
$$

namely

$$
P_{B_{1} \ldots B_{n}}^{A_{1} \ldots A_{n}}=\sum_{i=1}^{N} \iota_{i}^{A_{1} \ldots A_{n}} \iota_{B_{1} \ldots B_{n}}^{i *}
$$

where $\iota^{1 *}, \ldots, \iota^{N *}$ is the dual basis. In particular, one can use an orthonormal basis, and then

$$
\iota^{i *}=\iota_{i}^{\dagger} \text {. }
$$

The $3 j, 6 j, 10 j$ and $15 j$ symbols emerging in the context of the BC and EPRL intertwiners are related to specific choice of basis in the valency 4 of the spin-networks and to the 4 -tetrahedral spin-foams. The advantage of our notation is the independence of the choice of basis. 


\section{The injectivity of $\mathcal{I} \mapsto \iota_{\mathrm{EPRL}}(\mathcal{I})$ in the case $\gamma \geq 1$}

As one could see above, the EPRL spin-network $\mathrm{SU}(2) \times \mathrm{SU}(2)$ intertwiners $\iota_{\mathrm{EPRL}}(\mathcal{I})$ are labelled by the $\mathrm{SU}(2)$ intertwiners $\mathcal{I}$. However, we have not said anything about the kernel of the map

$$
\mathcal{I} \mapsto \iota_{\mathrm{EPRL}}(\mathcal{I})
$$

We will show now that this map is injective in the case

$$
k_{I}=j_{I}^{+}-j_{I}^{-},
$$

provided $j_{1}^{-}+\ldots+j_{n}^{-} \in \mathbb{N}$.

Suppose we have found some $\mathcal{I}_{0}$, such that

$$
\iota_{\mathrm{EPRL}}\left(\mathcal{I}_{0}\right)=0,
$$

that is

$$
P^{+A_{1}^{+} \ldots A_{n}^{+}} c_{1}^{+\ldots D_{n}^{+}} c_{1}^{D_{1}^{+} A_{1}^{-}}{ }_{B_{1}} \ldots c_{n}{ }^{D_{n}^{+} A_{n}^{-}}{ }_{B_{n}} \mathcal{I}_{0} B_{1} \ldots B_{n}=0 .
$$

Then, any contraction of the left-hand side also vanishes, in particular

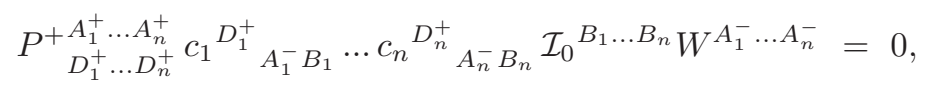

where we lowered the indices $A_{I}^{-}$with the $\epsilon_{j_{I}^{-}}$bilinear forms and took any $0 \neq W \in \operatorname{Inv}\left(\rho_{j_{1}^{-}} \otimes \ldots \otimes \rho_{j_{n}^{-}}\right)$. Such $W$ exists iff $j_{1}^{-}+\ldots+j_{n}^{-} \in \mathbb{N}$. We will show now, that the last equality can not be true unless $\mathcal{I}_{0}=0$ itself.

First, let us realize some property of the spin composition map defined given two half integers $k$ and $j$,

$$
c_{(k, j)}: \mathcal{H}_{k} \otimes \mathcal{H}_{j} \rightarrow \mathcal{H}_{k+j}
$$

intertwining the corresponding $\mathrm{SU}(2)$ representations. In our notation the map is given by the intertwiners $c^{A}{ }_{B C}$, namely

$$
\mathcal{H}_{k} \otimes \mathcal{H}_{j} \ni K^{B C} \mapsto K^{B C} c^{A}{ }_{B C} \in \mathcal{H}_{k+j}
$$

The key property is that the map can not annihilate a nonzero simple tensor $K^{B C}=\mathcal{I}^{B} W^{C}$

$$
\mathcal{I}^{B} W^{C} c_{B C}^{A}=0 \quad \Leftrightarrow \mathcal{I}^{B} W^{C}=0
$$

To see that this is really true, view each of the Hilbert spaces $\mathcal{H}_{l}, l \in \frac{1}{2} \mathbb{N}$ as the symmetric part of the tensor product $\mathcal{H}_{\frac{1}{2}} \otimes \ldots \otimes \mathcal{H}_{\frac{1}{2}}$, introduce an orthonormal basis $|0\rangle,|1\rangle \in \mathcal{H}_{\frac{1}{2}}$ and use it to define a basis $v_{0}^{(l)}, \ldots, v_{2 l}^{(l)} \in \mathcal{H}_{l}$,

$$
\begin{aligned}
& v_{0}^{(l)}:=|0\rangle \otimes \ldots \otimes|0\rangle, \\
& v_{1}^{(l)}:=\operatorname{Sym}|0\rangle \otimes \ldots \otimes|0\rangle \otimes|1\rangle, \\
& \ldots \\
& v_{2 l}^{(l)}:=|1\rangle \otimes \ldots \otimes|1\rangle,
\end{aligned}
$$

where

$$
\operatorname{Sym}: \mathcal{H}_{\frac{1}{2}} \otimes \ldots \mathcal{H}_{\frac{1}{2}} \rightarrow \mathcal{H}_{\frac{1}{2}} \otimes \ldots \mathcal{H}_{\frac{1}{2}}
$$

denotes the projection on the subspace of symmetric tensors. The spin composition map in this basis reads

$$
c_{(k, j)}: v_{m}^{(k)} \otimes v_{m^{\prime}}^{(j)} \mapsto v_{m+m^{\prime}}^{(k+j)} .
$$


Hence, the product of two general elements is mapped in the following way

$$
\sum_{m=m_{1}}^{2 k} \mathcal{I}^{\prime m} v_{m}^{(k)} \otimes \sum_{m^{\prime}=m_{1}^{\prime}}^{2 j} W^{\prime m^{\prime}} v_{m^{\prime}}^{(j)} \mapsto \mathcal{I}^{\prime m_{1}} W^{\prime m_{1}^{\prime}} v_{m_{1}+m_{1}^{\prime}}^{(k+j)}+\sum_{M>m_{1}+m_{1}^{\prime}} \alpha^{M} v_{M}^{(k+j)} \neq 0
$$

where $\mathcal{I}^{m_{1}}$ and $W^{m_{1}^{\prime}}$ are the first non-vanishing components. The result can not be zero, because the first term on the right-hand side is nonzero, and is linearly independent of the remaining terms.

Secondly, we generalize the statement (4.46) to the following one:

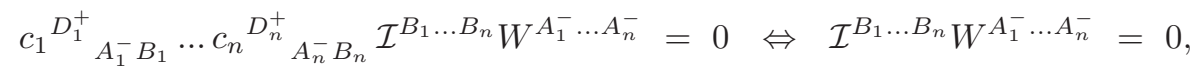

for arbitrary $\mathcal{I} \in \mathcal{H}_{k_{1}} \otimes \ldots \otimes \mathcal{H}_{k_{n}}$ and $W \in \mathcal{H}_{j_{1}^{-}} \otimes \ldots \otimes \mathcal{H}_{j_{n}^{-}}$. In the proof we will use a calculation similar to that of (4.48), with the difference that now the coefficients in (4.48) take values in the $n-1$ valent tensor products. Specifically, the left-hand side of the first equality in (4.49) is the result of the map

$$
c_{\left(k_{1}, j_{1}^{-}\right)} \otimes \ldots \otimes c_{\left(k_{n}, j_{n}^{-}\right)}: \mathcal{H}_{k_{1}} \otimes \ldots \otimes \mathcal{H}_{k_{n}} \otimes \mathcal{H}_{j_{1}^{-}} \otimes \ldots \otimes \mathcal{H}_{j_{n}^{-}} \rightarrow \mathcal{H}_{k_{1}+j_{1}^{-}} \otimes \ldots \otimes \mathcal{H}_{k_{n}+j_{n}^{-}},
$$

defined by (4.44), and applied to given $\mathcal{I}^{\prime}$ and $W^{\prime}$. By analogy to (4.48), we can write

$$
\mathcal{I}=\sum_{m=m_{1}}^{2 k_{1}} v_{m}^{\left(k_{1}\right)} \otimes \mathcal{I}^{m}, \quad W=\sum_{m^{-}=m_{1}^{-}}^{2 j_{1}^{-}} v_{m^{-}}^{\left(j_{-}^{-}\right)} \otimes W^{m^{-}}
$$

where $\mathcal{I}^{m_{1}}$ is the first nonvanishing $\mathcal{H}_{k_{2}} \otimes \ldots \otimes \mathcal{H}_{k_{n}}$ valued component of $\mathcal{I}$ and $W^{m_{1}^{-}}$is the first nonvanishing $\mathcal{H}_{j_{2}^{-}} \otimes \ldots \otimes \mathcal{H}_{j_{n}^{-}}$valued component of $W$. Now we apply the map (4.50),

$$
\begin{aligned}
c_{\left(k_{1}, j_{1}^{-}\right)} \otimes \ldots \otimes c_{\left(k_{n}, j_{n}^{-}\right)} & \left(\sum_{m=m_{1}}^{2 k_{1}} v_{m}^{\left(k_{1}\right)} \otimes \mathcal{I}^{m} \otimes \sum_{m^{-}=m_{1}^{-}}^{2 j_{1}^{-}} v_{m^{-}}^{\left(j_{1}^{-}\right)} \otimes W^{m^{-}}\right) \\
= & v_{m_{1}+m_{1}^{-}}^{\left(k_{1}+j_{1}^{-}\right)} \otimes\left(c_{\left(k_{2}, j_{2}^{-}\right)} \otimes \ldots \otimes c_{\left(k_{n}, j_{n}^{-}\right)}\left(\mathcal{I}^{m_{1}} \otimes W^{m_{1}^{-}}\right)\right)+ \\
& +\sum_{M>m_{1}+m_{1}^{-}} v_{M}^{\left(k_{1}+j_{1}^{-}\right)} \otimes \alpha^{M} .
\end{aligned}
$$

The first term on the right-hand side is linearly independent of the others, hence if it were nonzero so would be the right-hand side. But it is nonzero provided (4.49) holds for $n$ replaced by $n-1$. Since (4.49) is true for $n=1$, the mathematical induction does the rest of the job.

Finally, we notice that if

$$
\mathcal{I}=\mathcal{I}_{0} \in \operatorname{Inv}\left(\rho_{k_{1}} \otimes \ldots, \otimes \rho_{k_{n}}\right)
$$

and

$$
W \in \operatorname{Inv}\left(\rho_{j_{1}^{-}} \otimes \ldots, \otimes \rho_{j_{n}^{-}}\right)
$$

then the tensor

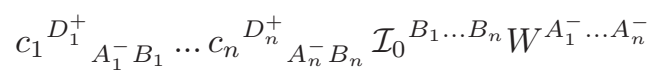

defines an element of $\operatorname{Inv}\left(\rho_{j_{1}^{+}} \otimes \ldots, \otimes \rho_{j_{n}^{+}}\right)$, that is,

$$
c_{\left(k_{1}, j_{1}^{-}\right)} \otimes \ldots \otimes c_{\left(k_{n}, j_{n}^{-}\right)}\left(\mathcal{I}_{0} \otimes W\right) \in \operatorname{Inv}\left(\rho_{j_{1}^{+}} \otimes \ldots, \otimes \rho_{j_{n}^{+}}\right) .
$$

Hence the projection $P^{+}$in (4.42) acts as the identity,

$$
P^{+} c_{\left(k_{1}, j_{1}^{-}\right)} \otimes \ldots \otimes c_{\left(k_{n}, j_{n}^{-}\right)}\left(\mathcal{I}_{0} \otimes W\right)=c_{\left(k_{1}, j_{1}^{-}\right)} \otimes \ldots \otimes c_{\left(k_{n}, j_{n}^{-}\right)}\left(\mathcal{I}_{0} \otimes W\right),
$$

hence

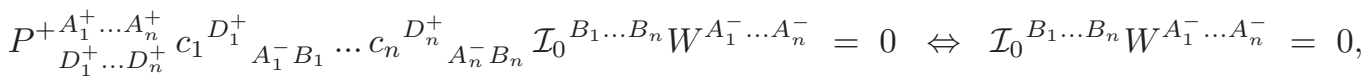

but $W$ is arbitrary in (4.42), hence

$$
\mathcal{I}_{0}=0 \text {. }
$$




\section{DISCUSSION}

\section{A. Summary}

The conclusion coming from our work is that we do not have to reformulate LQG in terms of the piecewise linear category and triangulations to match it with the EPRL SFM. The generalized spinfoam framework is compatible with the original LQG framework and accommodates all the spinnetwork states and the diffeomorphism covariance. Our generalization goes in two directions. The first one is from spin-foams defined on simplicial complexes to spin-foams defined on the arbitrary linear 2-cell complexes (see also [13]). The main tools of the SF models needed to describe 4dimensional spacetime are available: spin-foam, boundary spin-network, characterization of vertex, vertex amplitude, the scheme of the SF models of 4-dim gravity, the EPRL intertwiners.

The second direction is from abstract spin-foams to embedded spin-foams, histories of the embedded spin-network 2 For example the notion of knots and links is again available in that framework. One may for example consider a spin-foam history which turns an unknotted embedded circle into a knotted circle.

The most important result is the characterization of a vertex of a generalized spin-foam. The structure of each vertex can be completely encoded in a spin-network induced locally on the boundary of the vertex neighbourhood. The spin-network is used for the natural generalization of the vertex amplitude used in the simplicial spin-foam models. The characterization of the vertices leads to a general construction of a general spin-foam. The set of all the possible vertices is given by the set of the spin-networks and and set of all spin-foams can be obtained by gluing the vertices and the "static" spin-foams.

We have also proved the $1-1$ correspondence between the EPRL $\mathrm{SU}(2) \times \mathrm{SU}(2)$ intertwiners and all the $\mathrm{SU}(2)$ intertwiners in the case of the Immirzi-Barbero parameter $|\gamma| \geq 1$ and non-trivial co-domain of EPRL map.

In the literature, the $3 j, 6 j, 10 j$ and $15 j$ symbols are used extensively in the context of the BC and EPRL intertwiners and amplitudes. From our point of view, they are just related to a specific choice of the basis in the space of intertwiners valid in the case of the simplicial spin-foams and spin-networks. Our approach is basis independent.

\section{B. The limits $\gamma= \pm \infty, \pm 1,0$}

A subject that deserves an individual discussion are the limits of the EPRL model as $\gamma \rightarrow$ $0, \pm 1, \pm \infty$. Interestingly, all those limits are mathematically well defined, although the model seems to be losing its key properties.

The best established limit is

$$
\gamma \rightarrow \pm \infty
$$

That limit exists at each level: the Holst action converges to the Palatini action, $j^{+}=j^{-}, k=0$, the EPRL intertwiner converges to the BC intertwiner, all the EPRL derivation converges to a finite limit. The only surprise is the discontinuity in the number of the degrees of freedom. The BC intertwiner space has 0 or 1 dimension per vertex and has been proven to insufficiently accommodate all the gravitational degrees of freedom.

The limit that can not be extended to the entire derivation although the Holst action does converge perfectly well to the self dual action is

$$
\gamma= \pm 1
$$

However the EPRL intertwiner has a limit in that case:

$$
j^{\mp}=0, k=j^{ \pm},
$$

\footnotetext{
2 As a matter of fact the simplicial spin-foams considered in [4] were also embedded, however they did not use all the diversity given by the embeddings, since they were restricted to triangulations.
} 
and moreover

$$
\iota_{\mathrm{EPRL}}(\mathcal{I})=\mathcal{I}
$$

where $\mathcal{I}$ is an arbitrary $\mathrm{SU}(2)$ intertwiner, and the amplitude turns into the $\mathrm{SU}(2) \mathrm{BF}$ amplitude. So the limit theory is the $\mathrm{SU}(2) \mathrm{BF}$ theory. That is very strange, taking into account that the self dual action still defines the same Einstein's (Euclidean) gravity.

The limit in which the Holst action is no longer equivalent to the Palatini action and (upon the rescaling by $\gamma$ ) defines a topological theory is

$$
\gamma=0
$$

Then

$$
j^{+}=j^{-}, \quad k=j^{+}+j^{-}
$$

but, quite surprisingly, the EPRL theory does not resemble a topological theory at all.

Acknowledgements We would like to thank John Barrett, Jonathan Engle for coming to Warsaw and delivering lectures on the SFM. JL acknowledges the conversations with Laurent Freidel, and exchange of e-mails with John Baez, Carlo Rovelli, Roberto Pereira and Etera Livine. MK would like to thank Jonathan Engle from Albert Einstein Institute in Potsdam and Carlo Rovelli, Matteo Smerlak from Centre de Physique Théorique de Luminy for discussions and hospitality during his visits at their institutes. The work was partially supported by the Polish Ministerstwo Nauki i Szkolnictwa Wyzszego grants 182/NQGG/ 2008/0, 2007-2010 research project N202 n081 32/1844, the National Science Foundation (NSF) grant PHY-0456913, by the Foundation for Polish Science grant Master and a Travel Grant from the QG research networking programme of the European Science Foundation.

[1] Thiemann T (2007), Introduction to Modern Canonical Quantum General Relativity (Cambridge University Press, Cambridge)

Rovelli C (2004), Loop quantum gravity, (Cambridge University Press, Cambridge)

Ashtekar A and Lewandowski J (2004), Background independent quantum gravity: A status report, Class. Quant. Grav. 21 R53, (Preprint gr-qc/0404018)

Han M, Huang W, Ma Y (2007) Fundamental structure of loop quantum gravity, Int. J. Mod. Phys. D16, pp. 1397-1474

[2] Reisenberger MP (1994) World sheet formulations of gauge theories and gravity Preprint gr-qc/9412035

Reisenberger MP, Rovelli C (1997), "Sum over surfaces" form of loop quantum gravity , Phys.Rev. D56 3490-3508 (Preprint gr-qc/9612035)

[3] Markopoulou F (1997), Dual formulation of spin-network evolution, gr-qc/9704013

[4] Baez J, (2000) An introduction to Spinfoam Models of BF Theory and Quantum Gravity, Lect.Notes Phys. 543 25-94 (Preprint gr-qc/9905087v1)

[5] Perez A (2003), Spinfoam models for Quantum Gravity, Class.Quant.Grav. 20 R43 (Preprint gr-qc/0301113v2)

[6] Engle J, Livine E, Pereira R, Rovelli C (2008), LQG vertex with finite Immirzi parameter, Nucl.Phys. B799:136-149 (Preprint gr-qc/0711.0146v2)

[7] Noui K, Perez A (2005) Three dimensional loop quantum gravity: physical scalar product and spinfoam models, Class. Quant. Grav. 22, pp 1739-1762

[8] Barrett JW, Crane L (1998), Relativistic spin-networks and quantum gravity, J.Math.Phys. 39 32963302 (Preprint gr-qc/9709028)

[9] Alesci E, Rovelli C (2007), The complete LQG propagator I. Difficulties with the Barrett-Crane vertex, Phys.Rev. D76:104012, (Preprint gr-qc/0708.0883)

[10] Yetter D (1998), Generalized Barrett-Crane vertices and invariants of embedded graphs, math/9801131 Barrett JW (1998), The classical evaluation of relativistic spin net- works, Adv.Theor.Math.Phys.2:593-60 (Preprint math/9803063)

[11] Reisenberger MP (1998), On relativistic spin network vertices, J.Math.Phys. 40 (1999) 2046-2054 (Preprint gr-qc/9809067 1 )

[12] Oeckl R (2002), Renormalization of Discrete Models without Background,Nucl.Phys. B657 (2003) 107-138, (Preprint gr-qc/0212047v2 ) 
[13] Reisenberger MP (1997), A left-handed simplicial action for euclidean general relativity, Class. Quant. Gravity 141753 (Preprint gr-qc/9609002)

[14] Engle J, Pereira R and Rovelli C (2008), Flipped spinfoam vertex and loop gravity, Nucl.Phys. B798:251-290 (Preprint gr-qc/0708.1236v1)

Livine ER, Speziale S (2007), A new spinfoam vertex for quantum gravity,Phys.Rev. D76:084028 (Preprint gr-qc/0705.0674v2)

[15] Freidel L, Krasnov K (2008), A New Spin Foam Model for 4d Gravity, Class.Quant.Grav. 25:125018 (Preprint gr-qc/0708.1595v2)

[16] Engle J, Pereira R (2008), Coherent states, constraint classes, and area operators in the new spin-foam models, Class.Quant.Grav. 25:105010

[17] Ashtekar A, Baez J, Corichi A and Krasnov K (1998), Quantum Geometry and Black Hole Entropy Phys. Rev. Lett. 80 904-907 (Preprint arXiv:gr-qc/9710007)

Ashtekar A, Baez J and Krasnov K (2000) Quantum Geometry of Isolated Horizons and Black Hole Entropy Adv. Theor.Math.Phys. 4 1-94 (Preprint arXiv:gr-qc/0005126)

Domagała M, Lewandowski J (2004), Black hole entropy from Quantum Geometry Class.Quant.Grav. 21 5233-5244 (Preprint arXiv:gr-qc/0407051)

Meissner K (2004), Black hole entropy in loop quantum gravity Class.Quant.Grav. 21 5245-5252 (Preprint arXiv:gr-qc/0407052)

[18] Rovelli C, Smolin C (1995), Discreteness of area and volume in quantum gravity, Nucl. Phys. B 442 593 [1995 Erratum-ibid. B 456 753], (Preprint gr-qc/9411005)

Ashtekar A, Lewandowski J (1995), Differential Geometry on the Space of Connections via Graphs and Projective Limits J. Geom. Phys. 17 191-230, (Preprint hep-th/9412073)

Ashtekar A and Lewandowski J (1997), Quantum theory of geometry. I: Area operators,.Class. Quant. Grav. 14 A55, (Preprint gr-qc/9602046)

Ashtekar A and Lewandowski J (1998), Quantum theory of geometry. II: Volume operators,.Adv. Theor. Math. Phys. 1 388, (Preprint gr-qc/9711031)

Thiemann T (1998), A length operator for canonical quantum gravity J. Math. Phys. 39 3372, (Preprint gr-qc/9606092)

[19] Bahr B, Dittrich B (2009), Regge calculus from a new angle Preprint gr-qc/0907.4325

Bahr B, Dittrich B (2009), Improved and Perfect Actions in Discrete Gravity Preprint arXiv:0907.4323

[20] Conrady F, Freidel L (2009) Quantum geometry from phase space reduction Preprint arXiv:0902.0351

[21] Engle J (2008), Piecewise linear loop quantum gravity, gr-qc/0812.1270v1

[22] Zapata J A (2002) Continuum spin-foam model for 3d gravity J. Math. Phys. 43, pp. 5612-5623 (Preprint: gr-qc/0205037)

[23] Rourke C and Sanderson B (1972), Introduction to Piecewise-Linear Topology, (Springer Verlag, Berlin)

Lurie J, Topics in Geometric Topology, http://math.mit.edu/ lurie/937.html

[24] Bianchi E, Modesto L, Rovelli C,Speziale S (2006), Graviton propagator in loop quantum gravity Class.Quant.Grav. 23 6989-7028 (Preprint gr-qc/0604044)

Alesci E, Rovelli C (2007) The complete LQG propagator: II. Asymptotic behavior of the vertex Phys. Rev. D77, pp. 044024, Preprint: arXiv:0711.1284

Alesci E, Bianchi E, Rovelli C (2008) LQG propagator: III. The new vertex Preprint.arXiv:0812.5018 Mamone D, Rovelli C (2009) Second-order amplitudes in loop quantum gravity Preprint: arXiv:0904.3730

Bianchi E, Magliaro E, Perini C (2009), LQG propagator from the new spin foams, Nuclear Physics B 822 pp. 245-269, (Preprint arXiv:0905.4082)

[25] Oeckl R (2002), Generalized Lattice Gauge Theory, Spin Foams and State Sum Invariants, J. Geom. Phys. 46 (2003) 308-354, (Preprint hep-th/0110259)

Oeckl R (2005), Discrete gauge theory: From Lattices to TQFT, Imperial College Press

[26] Rovelli C (1991), Quantum Mechanics without Time: A Model Phys. Rev. D 422638

Rovelli C (1991), Time in Quantum Mechanics: Physics beyond the Schroedinger Regime Phys. Rev. D 43442 .

[27] Holst S (1996), Barbero's hamiltonian derived from a generalised Hilbert-Palatini action, Phys. Rev. D53 5966-5969,

[28] Baratin A, Flori C, Thiemann T (2008), The Holst Spin Foam Model via Cubulations, arXiv:0812.4055v2

[29] Barbero F (1996), Real Ashtekar variables for Lorentzian signature space-times, Phys. Rev. D51 55075510

Immirzi G (1997), Quantum gravity and Regge calculus, Nucl. Phys. Proc. Suppl. 576572 